\section{FT-IR, FT-Raman and UV-Visible Spectral Analysis on (E)-N'-(thiophen-2-ylmethylene) Nicotinohydrazide}

\section{Abstract}

Vibrational analysis of the (E)- $\mathrm{N}^{\prime}$-(thiophen-2-ylmethylene) nicotinohydrazide (T2CNH) compound was carried out in solid phase using FTIR and FT-Raman spectroscopic techniques in the ranges: $400-4000 \mathrm{~cm}^{-1}$ and $100-4000 \mathrm{~cm}^{-1}$, respectively. The molecular geometries and harmonic vibrational frequencies were calculated using DFT/6-311++G(d,p) basis set. A detailed interpretation of the IR and Raman spectra, based on the total energy distribution (TED) of the normal modes. The bond parameters such as bond lengths, bond angles and dihedral angles were calculated at the same level of theory. The natural bonding orbital (NBO) study reveals that inter and intra-molecular charge transfer of the molecule. The electronic transition was studied using UV-Vis spectrum. The NLO, band gap energy, MEP map, Mulliken atomic charges were calculated using the same level of basis set. In addition the thermodymanic properties were also calculated.

Keywords: FT-IR; FT-Raman; TED; NBO; T2CNH

Received: December 21, 2016; Accepted: January 02, 2017; Published: January 09, 2017

\section{Introduction}

Generally, Pyridine ring is a heterocyclic organic compound with the chemical formula $\mathrm{C}_{5} \mathrm{H}_{5} \mathrm{~N}$. Pyridine is structurally related to benzene, with one methine group $(=\mathrm{CH}-)$ replaced by a nitrogen atom. It occurs in many important compounds, including azines and the vitamins niacin and pyridoxal. The precursor of pyridine is used to agrochemicals, pharmaceuticals and is also an important solvent and reagent. Mostly, it is used in the in vitro synthesis of DNA, sulfa pyridine (a drug against bacterial and viral infections), antihistaminic drugs tripelennamine and mepyramine, as well as water repellents, bactericides, and herbicides. Some chemical compounds, although not synthesized from pyridine, contain its ring structure. They include B vitamins niacin and pyridoxal, an anti-tuberculosis drug isoniazide, nicotine and other nitrogencontaining plant products [1].

The ring of Thiophene and its derivatives have been reported to possess broad spectrum of biological properties including anti-inflammatory, analgesic, antidepressant, antimicrobial and anticonvulsant activities [2-4]. Antiepileptic drugs (AEDs) like tiagabine, etizolam, brotizolam are containing thiophene moiety
Bharanidharan $\mathbf{S}^{1}$, Saleem $\mathrm{H}^{1}$, Subashchandrabose $\mathrm{S}^{2}$, Suresh $\mathrm{M}^{3}$ and

\section{Ramesh Babu N ${ }^{4}$}

1 Department of Physics, Annamalai University, Annamalainagar-608002, Tamil Nadu, India

2 Centre for Research and Development, PRIST University, Thanjavur-613403, Tamil Nadu, India

3 Department of Chemistry, College of Engineering, Guindy, Anna Universtiy, Chennai, 620 025, India

4 Dept. of Physics, M.I.E.T. Engineering College, Trichy-620007, Tamil Nadu, India

Corresponding author: Saleem H

saleem_h2001@yahoo.com

Department of Physics, Annamalai University, Tamil Nadu, India.

Tel: +919443879295

Citation: Bharanidharan S, Saleem $\mathrm{H}$, Subashchandrabose S, et al. FT-IR, FT-Raman and UV-Visible Spectral Analysis on (E)-N'(thiophen-2-ylmethylene) Nicotinohydrazide. Arch Chem Res. 2017, 1:2.

in their structures as active pharmacophore $[5,6]$. In addition, thiophene and its derivatives functionalized with the formyl group are versatile building blocks for the synthesis of donoracceptor substituted $\mathrm{p}$-conjugated systems for several optical applications.

The hydrazone group in the organic compound brings out several physical and chemical properties. The hydrazones are bearing the $>\mathrm{C}=\mathrm{N}-\mathrm{N}<$ which leads the molecule towards nucleophilic and electrophilic in nature. In the hydrazone moiety, the nitrogen atom behaves as nucleophilic and carbon atom behaves as nucleophilic as well as electrophilic in nature [7-9]. The benzohydrazide derivatives shows wide spectrum of 
biological activities such as antibacterial [10], antifungal [11] and antitubercular [12] activities.

Subashchandrabose et al. [13] recorded the FT-IR, FT-Raman and UV-Vis spectra for the molecule N1-N2-bis((pyridine-4-I) methylene)benzene-1,2-diamine. The observed FT-IR and FTRaman spectral values were compared with the calculated wave numbers. For the prediction of accurate vibrational assignments TED analysis was performed using SQM method. The bond lengths and bond angles of stable conformer were correlated well with the experimental values. The hyperconjugative interaction and charge delocalization around the bonds were studied using NBO analysis. Band gap energy was also determined.

Quantum chemical calculations of energies, geometrical structure and vibrational wavenumber of 1,2-bis(3-methoxy-4hydroxybenzylidene)hydrazine were carried out by Subramanian et al. [14] using DFT method with $6-31 \mathrm{G}(\mathrm{d})$ as basis set. The optimized geometrical parameters obtained by DFT calculations are in good agreement with single crystal XRD data. The vibrational spectral data were obtained from FT-IR and FT-Raman spectra are assigned based on the results of the theoretical calculations in solid phase.

From Literature survey reveals that the vibrational analysis of (E)- $\mathrm{N}^{\prime}$-(thiophen-2-ylmethylene) nicotinohydrazide (T2CNH) has not yet been reported. The T2CNH molecule was synthesized and its structural characterization was calculated by B3LYP/6$311++G(d, p)$ basis set. The spectral investigation such as FT-IR, FTRaman and UV-Vis spectra were recorded. The observed spectral results were compared with the computed wavenumber. The vibrational assignments of the title molecule were carried out with the help of TED. The First order hyperpolarizability, HomoLumo energy gap was calculated and furthermore, the MEP and thermodynamic properties were also calculated.

\section{Computational Details}

The entire theoretical calculations were performed DFT method at B3LYP/6-311++G(d,p) basis set to using Gaussian 03W [15] program package, invoking gradient geometry optimization $[15,16]$. The geometrical parameters were used in the vibrational frequency calculations at the same level to characterize all the stationary points as minima. The vibrationally averaged nuclear positions of $\mathrm{T} 2 \mathrm{CNH}$ were used for harmonic vibrational frequency calculations resulting in IR and Raman frequencies together with intensities and Raman depolarization ratios. The vibrational modes were assigned on the basis of TED analysis using VEDA4 program package [17]. The Raman activity was calculated by using Gaussian 03W package and the activity was transformed into Raman intensity using Raint program [18] by the expression:

$I_{i}=10^{-12} \times\left(v_{o}-v_{i}\right)^{4} \times \frac{1}{v_{i}} \times R A_{i}$

Where $\mathrm{li}$ is the Raman intensity, RAi is the Raman scattering activities, $v i$ is the wavenumber of the normal modes and $v_{0}$ denotes the wavenumber of the excitation laser [19].

\section{Experimental Details}

\section{Synthesis procedure}

A mixture of Thiophene-2-carboxaldehyde $(2.1 \mathrm{~mL}, 0.01 \mathrm{~mol})$ and nicotinic acid hydrazide $(1.37 \mathrm{~g}, 0.01 \mathrm{~mol})$ in $5 \mathrm{~mL}$ of ethanol was stirred in the presence of 2 drops of concentrated $\mathrm{HCl}$ for one hour. The reaction mixture was maintained at room temperature and the colourless solid was obtained. The solid was separated and filtered under suction, washed with ice-cold water $(50 \mathrm{ml})$. The precipitate was washed with water and filtered and again washed with petroleum ether (40-60\%) and dried over in a vacuum desicator then the product was recrystallized from hot ethanol.

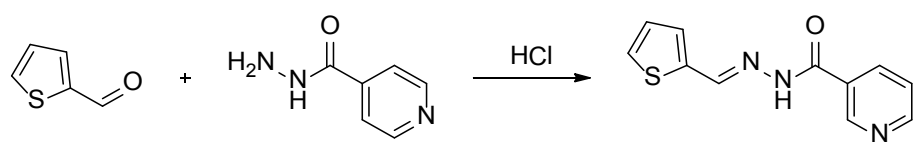

\section{Results and Discussion}

\section{Molecular Geometry}

The T2CNH molecule was optimized by B3LYP/6-311++G(d,p) level of basis set. This molecule consists of thiophene and pyridine rings are fused by hydrazone linkage. The calculated bond length of $\mathrm{C}_{2}-\mathrm{S}_{1}$ is expected to be shorter than the $\mathrm{C}_{5}-\mathrm{S}_{1}$ bond length, since the hydrozone moiety is attached with $C_{2}$ atom and their corresponding bond distances are 1.750 and 1.728 $\AA$. The bond lengths of $C_{2}=C_{3}, C_{3}-C_{4}, C_{4}=C_{5}$ are 1.377, 1.418, $1.369 \AA$ and the bond angles of C2-C3-C4, C3-C4-C5 are $113.02^{\circ}$, $113.03^{\circ}$. The bond angles $\mathrm{S}_{1}-\mathrm{C}_{2}-\mathrm{C}_{3}$ and $\mathrm{S}_{1}-\mathrm{C}_{5}-\mathrm{C}_{4}$ are differ by $\sim 1^{\circ}$ which is due to the shortening of bond distance between $S_{1}$ and $C_{2}$ atoms. These values are particularly in good agreement with the values of anti thiophen-2-aldehyde [20] and thiophen2-carbohydrazide [21]. Furthermore, these values are also find support from literature [22]. The dihedral angles of $\mathrm{S}_{1}-\mathrm{C}_{2}-\mathrm{C}_{9}-\mathrm{N}_{11} /$ $\mathrm{S}_{1}-\mathrm{C}_{2}-\mathrm{C}_{9}-\mathrm{H}_{1} \mathrm{O}$ and $\mathrm{C}_{3}-\mathrm{C}_{2}-\mathrm{C}_{9}-\mathrm{N}_{11} / \mathrm{C}_{3}-\mathrm{C}_{2}-\mathrm{C}_{9}-\mathrm{H}_{10}$ are $-179.96^{\circ} /-0.07^{\circ}$ and $0.03^{\circ} / 179.93^{\circ}$, respectively, which shows that the thiophen and hydrozone moieties of $\mathrm{T} 2 \mathrm{CNH}$ are co-planar. Since there is a good conjugation between p-orbitals of all atoms of thiophen and hydrazone moieties. Most of the calculated geometrical parameters are find support from single crystal X-ray diffraction values $[23,24]$. It should be mentioned that there is no significant difference between the geometrical parameters of the hydrozone and pyridine moieties $[23,24]$. The geometrical parameters and optimized structure of the $\mathrm{T} 2 \mathrm{CNH}$ molecule are presented in Table 1 and Figure 1, respectively.

\section{Vibrational Assignments}

The $\mathrm{T} 2 \mathrm{CNH}$ molecule belongs to $\mathrm{C}_{1}$ point group symmetry. It consists of 25 atoms which undergoes 69 normal modes of vibrations. In which 47 modes of vibrations are in-plane and 22 are out-of-plane bending vibrations and all of them are IR and Raman active [25]. The calculated and observed vibrational wavenumbers using DFT/B3LYP/6-311++G(d,p) basis set, along with their relative intensities are given in Table 2 . The total energy distribution (TED) was calculated using SQM program [26]. The 
Table 1 The optimized bond parameters of $\mathrm{T}_{2} \mathrm{CNH}$.

\begin{tabular}{|c|c|c|}
\hline Bond Parametres & \multirow{2}{*}{ B3LYP/6-311++G(d,p) } & \multirow{2}{*}{$\mathrm{XRD}^{\mathrm{a}, \mathrm{b}, \mathrm{c}}$} \\
\hline Bond Lengths (Å) & & \\
\hline $\mathrm{S} 1-\mathrm{C} 2$ & 1.750 & $1.728^{b}$ \\
\hline S1-C5 & 1.728 & $1.717^{\mathrm{a}}, 1.707^{\mathrm{b}}$ \\
\hline $\mathrm{C} 2-\mathrm{C} 3$ & 1.377 & $1.375^{\mathrm{a}}, 1.362^{\mathrm{b}}$ \\
\hline $\mathrm{C} 2-\mathrm{C} 9$ & 1.448 & \\
\hline $\mathrm{C} 3-\mathrm{C} 4$ & 1.418 & $1.431^{\mathrm{a}}, 1.392^{\mathrm{b}}$ \\
\hline $\mathrm{C} 3-\mathrm{H} 8$ & 1.081 & $1.114^{\mathrm{a}}$ \\
\hline $\mathrm{C} 4-\mathrm{C} 5$ & 1.369 & $1.345^{b}$ \\
\hline $\mathrm{C} 9-\mathrm{H} 10$ & 1.097 & $0.930^{c}$ \\
\hline C9-N11 & 1.282 & $1.266^{c}$ \\
\hline N11-N12 & 1.357 & $1.375^{\mathrm{c}}$ \\
\hline $\mathrm{N} 12-\mathrm{H} 13$ & 1.016 & $0.860^{c}$ \\
\hline $\mathrm{N} 12-\mathrm{C} 14$ & 1.385 & $1.354^{c}$ \\
\hline C14-O15 & 1.213 & $1.224^{c}$ \\
\hline $\mathrm{C} 16-\mathrm{C} 17$ & 1.399 & $1.384^{c}$ \\
\hline $\mathrm{C} 16-\mathrm{C} 18$ & 1.397 & $1.371^{\mathrm{c}}$ \\
\hline C17-N19 & 1.335 & $1.334^{c}$ \\
\hline $\mathrm{C} 17-\mathrm{H} 20$ & 1.087 & $0.930^{c}$ \\
\hline $\mathrm{C} 18-\mathrm{C} 21$ & 1.387 & $1.368^{\mathrm{c}}$ \\
\hline $\mathrm{C} 18-\mathrm{H} 22$ & 1.083 & $0.930^{c}$ \\
\hline N19-C23 & 1.335 & $1.337^{c}$ \\
\hline $\mathrm{C} 21-\mathrm{C} 23$ & 1.395 & $1.368^{c}$ \\
\hline $\mathrm{C} 23-\mathrm{H} 25$ & 1.086 & $0.930^{c}$ \\
\hline \multicolumn{3}{|l|}{ Bond Angles $\left({ }^{\circ}\right)$} \\
\hline $\mathrm{C2}-\mathrm{S} 1-\mathrm{C} 5$ & 91.57 & $92.00^{a}, 91.81^{b}$ \\
\hline $\mathrm{S} 1-\mathrm{C} 2-\mathrm{C} 3$ & 110.68 & $111.80^{\mathrm{a}}$ \\
\hline $\mathrm{C} 2-\mathrm{C} 3-\mathrm{C} 4$ & 113.02 & $112.20^{\mathrm{a}}, 114.72^{\mathrm{b}}$ \\
\hline $\mathrm{C} 2-\mathrm{C} 3-\mathrm{H} 8$ & 122.16 & $129.20^{\mathrm{a}}$ \\
\hline $\mathrm{C} 3-\mathrm{C} 4-\mathrm{C5}$ & 113.03 & $112.05^{b}$ \\
\hline S1-C5-C4 & 111.68 & $112.13^{b}$ \\
\hline N12-C14-C16 & 114.21 & $114.95^{c}$ \\
\hline O15-C14-C16 & 122.13 & $121.48^{c}$ \\
\hline C16-C17-N19 & 123.88 & $122.05^{c}$ \\
\hline $\mathrm{C} 16-\mathrm{C} 17-\mathrm{H} 20$ & 120.91 & $120.03^{c}$ \\
\hline $\mathrm{N} 19-\mathrm{C} 17-\mathrm{H} 20$ & 115.16 & $118.20^{c}$ \\
\hline $\mathrm{C} 16-\mathrm{C} 18-\mathrm{C} 21$ & 118.94 & $118.91^{c}$ \\
\hline $\mathrm{C} 16-\mathrm{C} 18-\mathrm{H} 22$ & 119.08 & $120.50^{c}$ \\
\hline $\mathrm{C} 21-\mathrm{C} 18-\mathrm{H} 22$ & 121.96 & $120.30^{c}$ \\
\hline C17-N19-C23 & 117.47 & $117.44^{c}$ \\
\hline $\mathrm{C} 18-\mathrm{C} 21-\mathrm{C} 23$ & 118.58 & $118.58^{c}$ \\
\hline $\mathrm{C} 18-\mathrm{C} 21-\mathrm{H} 24$ & 121.13 & $120.71^{c}$ \\
\hline $\mathrm{C} 23-\mathrm{C} 21-\mathrm{H} 24$ & 120.27 & $120.70^{c}$ \\
\hline N19-C23-C21 & 123.40 & $123.64^{c}$ \\
\hline \multicolumn{3}{|l|}{ Dihedral Angles $\left({ }^{\circ}\right)$} \\
\hline S1-C2-C9-H10 & -0.07 & \\
\hline S1-C2-C9-N11 & -179.96 & \\
\hline C3-C2-C9-H10 & 179.93 & \\
\hline C3-C2-C9-N11 & 0.03 & \\
\hline C2-C3-C4-C5 & 0.02 & \\
\hline $\mathrm{C} 2-\mathrm{C} 3-\mathrm{C} 4-\mathrm{H} 7$ & -179.98 & \\
\hline $\mathrm{H} 8-\mathrm{C} 3-\mathrm{C} 4-\mathrm{C} 5$ & 179.97 & \\
\hline $\mathrm{H} 8-\mathrm{C} 3-\mathrm{C} 4-\mathrm{H} 7$ & -0.03 & \\
\hline C3-C4-C5-S1 & -0.01 & \\
\hline C3-C4-C5-H6 & -179.99 & \\
\hline
\end{tabular}

\begin{tabular}{|c|c|c|}
\hline H7-C4-C5-S1 & 179.99 \\
\hline H7-C4-C5-H6 & 0.01 \\
\hline C2-C9-N11-N12 & 179.45 \\
\hline H10-C9-N11-N12 & -0.43 \\
\hline C9-N11-N12-H13 & 1.93 \\
\hline N11-N12-C14-O15 & 175.56 \\
\hline N11-N12-C14-C16 & -2.82 \\
\hline H13-N12-C14-O15 & 177.89 \\
\hline H13-N12-C14-C16 & 170.80 \\
\hline N12-C14-C16-C17 & -8.47 \\
\hline N12-C14-C16-C18 & -28.41 \\
\hline O15-C14-C16-C17 & 154.27 \\
\hline O15-C14-C16-C18 & 152.29 \\
\hline C14-C16-C17-N19 & -25.01 \\
\hline C14-C16-C17-H20 & -178.88 \\
\hline C18-C16-C17-N19 & -0.97 \\
\hline
\end{tabular}

aBrathen O, Kveseth K, Nielsen KJ, Hagen K (1986) J Mol Struct 145: 45. 'Geiger DK, Geiger HC, Williams L, Noll BC (2012) Acta Cryst E 68 : 0420. 'Ramesh Babu N (2014) J Mol Struct 1072: 84-93.

combined vibrational spectra of $\mathrm{T} 2 \mathrm{CNH}$ molecule are shown in Figures 2 and 3.

\section{$\mathrm{N}-\mathrm{H}$ vibrations}

The $\mathrm{N}-\mathrm{H}$ stretching vibration appears in the region of 3300-3500 $\mathrm{cm}^{-1}$ [27]. In accordance with the above literature the $\mathrm{vN}-\mathrm{H}$ vibration has been observed at $3401 \mathrm{~cm}^{-1}$ in FTIR spectrum, whereas the harmonic wavenumber assigned at $3365 \mathrm{~cm}^{-1}$ (mode no: 1 ) and its TED value is $100 \%$ it should be noted here that, a small deviation between theoretical and experimental value is only due to intra-molecular charge transfer between amino and carbonyl group in hydrazone linkage. The harmonic wavenumbers (1508/ mode no: 14 and $488 \mathrm{~cm}^{-1} /$ mode no: 53 ) of $\beta \mathrm{N}-\mathrm{H}$ and $\mathrm{\Gamma N}-\mathrm{H}$ modes were presented in Table 2 are found to be in good agreement with literature [28] data 1476 and $535 \mathrm{~cm}^{-1}$ as well as with the structurally related molecule. Mode no: 14 is in agreement with observed FT-Raman band at $1519 \mathrm{~cm}^{-1}$ and these assignments also find support from TED values ( $22 \%$ and $61 \%$ ).

\section{$\mathrm{C}-\mathrm{H}$ vibrations}

In aromatic compounds, the $\mathrm{vCH}, \mathrm{BCH}$ and $\mathrm{\Gamma CH}$ vibrations are expected to appear in the ranges of $3100-3000 \mathrm{~cm}^{-1}, 1400-1000$ $\mathrm{cm}^{-1}$ and $1000-750 \mathrm{~cm}^{-1}$, respectively $[29,30]$. In this study, the aromatic $\mathrm{C}-\mathrm{H}$ stretching vibration is observed at $3059 \mathrm{~cm}^{-1} / \mathrm{FT}-\mathrm{IR}$, whereas the calculated wavenumbers are in the range 3076-3016 $\mathrm{cm}^{-1}$ (mode nos. 5 and 9). The in-plane $\mathrm{C}-\mathrm{H}$ bending vibrations are assigned at 1425, $1290 \mathrm{~cm}^{-1}$ in FT-IR and 1429, 1300 \& 1195 $\mathrm{cm}^{-1}$ in FT-Raman spectra and their corresponding calculated wavenumbers are 1447, 1391, 1308, $1173 \mathrm{~cm}^{-1}$ (mode nos: 16, $18,21,25)$. The harmonic wavenumbers occur in the region of 974-808 $\mathrm{cm}^{-1}$ (mode nos: $34-36,42$ ) are assigned to $\mathrm{C}-\mathrm{H}$ out-ofplane bending modes, in which mode no: 35 is in agreement with observed wavenumber values: $955 \mathrm{~cm}^{-1}$ (FT-Raman) and $948 \mathrm{~cm}^{-1}$ (FT-IR). These assignments are in line with literature values [14] and also find support from TED Values. 


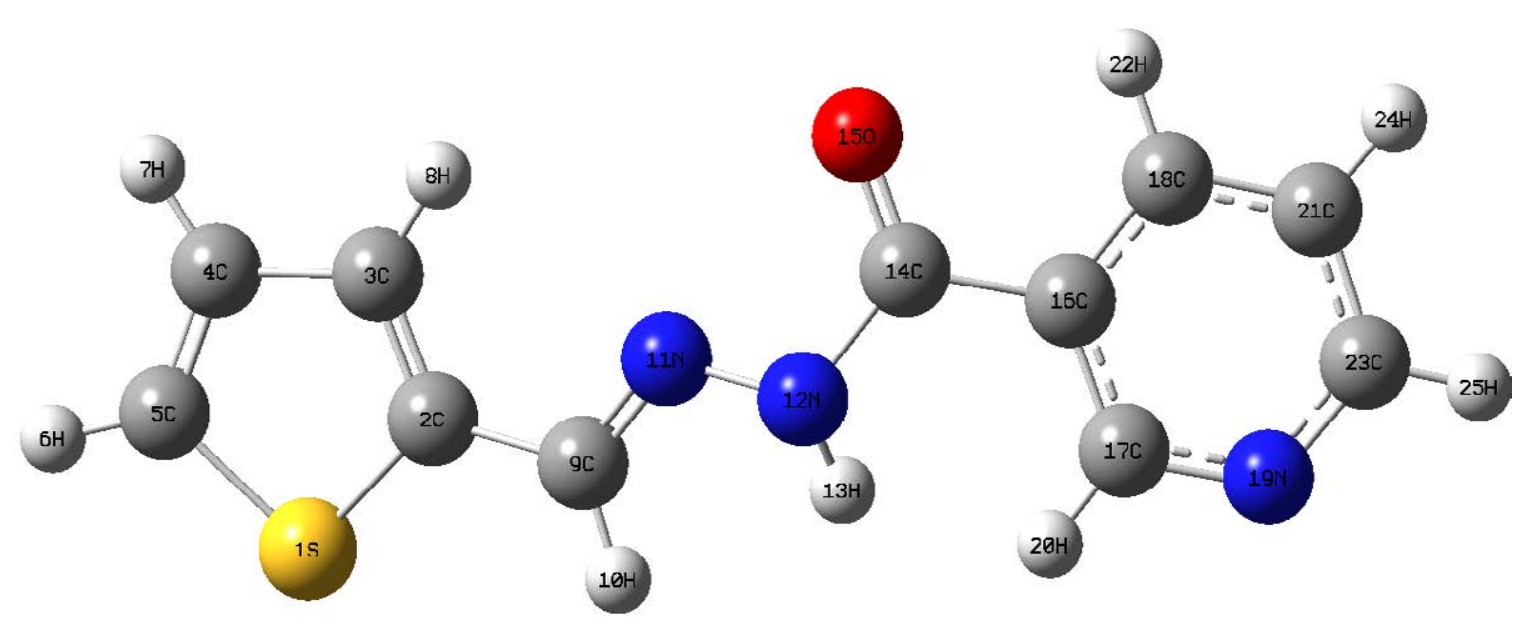

Figure 1 The Optimized structure of (E)-N'-(thiophen-2-ylmethylene) nicotinohydrazide (T2CNH).

Table 2 The experimental and calculated frequencies of $\mathrm{T}_{2} \mathrm{CNH}$ using B3LYP/6-311++G(d,p) level of basis set [harmonic frequencies $\left(\mathrm{cm}^{-1}\right)$, IR, Raman intensities $(\mathrm{Km} / \mathrm{mol})$, reduced masses (amu) and force constants $\left.\left(\mathrm{mdynA}^{\circ-1}\right)\right]$.

\begin{tabular}{|c|c|c|c|c|c|c|c|c|c|c|c|}
\hline \multirow{2}{*}{$\begin{array}{l}\text { Mode } \\
\text { No }\end{array}$} & \multicolumn{2}{|c|}{$\begin{array}{l}\text { Calculated } \\
\text { Frequencies } \\
\quad\left(\mathrm{cm}^{-1}\right)\end{array}$} & \multicolumn{2}{|c|}{$\begin{array}{c}\text { Observed } \\
\text { Frequencies } \mathrm{cm}^{-1} \text { ) }\end{array}$} & \multicolumn{2}{|c|}{ IR Intensity } & \multicolumn{2}{|c|}{ Raman Intensity } & \multirow{2}{*}{$\begin{array}{l}\text { Reduced } \\
\text { Masses }\end{array}$} & \multirow{2}{*}{$\begin{array}{l}\text { Force } \\
\text { Consts }\end{array}$} & \multirow[t]{2}{*}{ Vibrational assignments $\geq 10 \%$ (TED) $^{d}$} \\
\hline & $\begin{array}{l}\text { Un } \\
\text { Scaled }\end{array}$ & Scaled $^{\mathrm{a}}$ & FT-IR & FT-Raman & Abs. & Rel. $^{b}$ & Abs. & Rel. $^{c}$ & & & \\
\hline 1 & 3503 & 3365 & 3401 w & & 7.42 & 2.06 & 134.41 & 1.67 & 1.08 & 7.78 & $\mathrm{vN}_{12} \mathrm{H}_{13}(100)$ \\
\hline 2 & 3247 & 3120 & $3171 \mathrm{w}$ & & 1.23 & 0.34 & 101.27 & 1.26 & 1.10 & 6.82 & $\mathrm{vC}_{5} \mathrm{H}_{6}(94)$ \\
\hline 3 & 3223 & 3096 & & & 0.57 & 0.16 & 34.50 & 0.43 & 1.10 & 6.70 & $\mathrm{vC}_{3} \mathrm{H}_{8}(97)$ \\
\hline 4 & 3204 & 3078 & & $3079 w$ & 6.29 & 1.75 & 62.54 & 0.78 & 1.09 & 6.59 & $\mathrm{VC}_{4} \mathrm{H}_{7}(96)$ \\
\hline 5 & 3202 & 3076 & & & 7.00 & 1.94 & 72.48 & 0.90 & 1.10 & 6.62 & $v \mathrm{C}_{18} \mathrm{H}_{22}(99)$ \\
\hline 6 & 3187 & 3062 & $3059 w$ & & 9.14 & 2.54 & 84.20 & 1.05 & 1.09 & 6.53 & $v \mathrm{C}_{21} \mathrm{H}_{24}(95)$ \\
\hline 7 & 3156 & 3032 & & & 10.92 & 3.03 & 72.05 & 0.89 & 1.09 & 6.39 & $v \mathrm{C}_{23} \mathrm{H}_{25}(92)$ \\
\hline 8 & 3139 & 3016 & & & 29.24 & 8.12 & 29.47 & 0.37 & 1.09 & 6.32 & $v \mathrm{C}_{17} \mathrm{H}_{20}(99)$ \\
\hline 9 & 3019 & 2901 & & & 42.64 & 11.84 & 29.11 & 0.36 & 1.09 & 5.83 & $v \mathrm{CC}_{9} \mathrm{H}_{10}(100)$ \\
\hline 10 & 1757 & 1688 & $1673 \mathrm{~s}$ & $1670 w$ & 360.16 & 100.00 & 392.18 & 4.87 & 11.08 & 20.16 & $\mathrm{vO}_{15} \mathrm{C}_{14}(85)$ \\
\hline 11 & 1656 & 1591 & $1592 \mathrm{~s}$ & $1589 \mathrm{~s}$ & 15.73 & 4.37 & 8057.31 & 100.00 & 7.23 & 11.68 & $v N_{11} C_{9}(75)+\beta H_{10} C_{9} N_{11}(20)$ \\
\hline 12 & 1627 & 1563 & & & 32.79 & 9.10 & 1339.53 & 16.63 & 5.75 & 8.96 & $v C_{18} C_{21}(30)+v N_{19} C_{23}(30)+\beta C_{21} C_{23} N_{19}(25)$ \\
\hline 13 & 1605 & 1542 & 1549 w & & 6.21 & 1.73 & 25.40 & 0.32 & 5.21 & 7.91 & $v C_{21} C_{23}(31)+v C_{16} C_{18}(27)+\beta C_{17} N_{19} C_{23}(10)$ \\
\hline 14 & 1570 & 1508 & & $1519 w$ & 217.56 & 60.41 & 126.25 & 1.57 & 3.38 & 4.91 & $v C_{2} C_{3}(28)+\beta H_{13} N_{12} N_{11}(22)+v C_{4} C_{5}(13)+v C_{2} C_{9}(11)$ \\
\hline 15 & 1551 & 1490 & & & 202.85 & 56.32 & 2133.94 & 26.48 & 2.19 & 3.10 & $v C_{2} C_{3}(14)+\beta H_{13} N_{12} N_{11}(41)+v C_{4} C_{5}(12)$ \\
\hline 16 & 1506 & 1447 & $1425 \mathrm{~m}$ & $1429 \mathrm{~m}$ & 26.23 & 7.28 & 58.60 & 0.73 & 2.15 & 2.87 & $\mathrm{BH}_{24} \mathrm{C}_{21} \mathrm{C}_{23}(27)+\beta \mathrm{H}_{20} \mathrm{C}_{17} \mathrm{C}_{16}(25)$ \\
\hline 17 & 1459 & 1402 & & & 8.66 & 2.41 & 4817.87 & 59.80 & 4.83 & 6.07 & $v C_{2} C_{3}(13)+v C_{4} C_{5}(32)+\beta H_{7} C_{4} C_{5}(14)+v C_{3} C_{4}(15)$ \\
\hline 18 & 1448 & 1391 & & & 26.37 & 7.32 & 62.23 & 0.77 & 2.18 & 2.69 & $\mathrm{BH}_{25} \mathrm{C}_{23} \mathrm{~N}_{19}(43)+\mathrm{BC}_{16} \mathrm{C}_{17} \mathrm{~N}_{19}(23)$ \\
\hline 19 & 1390 & 1335 & & & 52.04 & 14.45 & 1478.54 & 18.35 & 1.75 & 1.99 & $v C_{4} C_{5}(13)+\beta H_{10} C_{9} N_{11}(38)+\beta H_{6} C_{5} C_{4}(13)$ \\
\hline 20 & 1387 & 1333 & & & 16.34 & 4.54 & 60.17 & 0.75 & 2.08 & 2.36 & $\mathrm{BH}_{10} \mathrm{C}_{9} \mathrm{~N}_{11}(25)+\mathrm{BH}_{6} \mathrm{C}_{5} \mathrm{C}_{4}(15)+v \mathrm{C}_{3} \mathrm{C}_{4}(18)$ \\
\hline 21 & 1362 & 1308 & $1290 \mathrm{~s}$ & $1300 \mathrm{~m}$ & 0.98 & 0.27 & 2.27 & 0.03 & 1.31 & 1.43 & $\mathrm{BH}_{20} \mathrm{C}_{17} \mathrm{C}_{16}(45)+\beta \mathrm{H}_{22} \mathrm{C}_{18} \mathrm{C}_{21}(23)$ \\
\hline 22 & 1291 & 1240 & & $1241 w$ & 19.71 & 5.47 & 141.74 & 1.76 & 7.28 & 7.15 & $\mathrm{vN}_{19} \mathrm{C}_{23}(43)+\mathrm{vC}_{16} \mathrm{C}_{18}(27)$ \\
\hline 23 & 1268 & 1219 & $1218 \mathrm{~m}$ & $1225 w$ & 94.23 & 26.16 & 45.69 & 0.57 & 1.54 & 1.46 & $\mathrm{BH}_{7} \mathrm{C}_{4} \mathrm{C}_{5}(23)+\mathrm{\beta H}_{8} \mathrm{C}_{3} \mathrm{C}_{4}(29)+\mathrm{\beta H}_{6} \mathrm{C}_{5} \mathrm{C}_{4}(13)$ \\
\hline 24 & 1265 & 1215 & & & 221.71 & 61.56 & 2432.18 & 30.19 & 2.39 & 2.25 & $v C_{14} C_{16}(22)$ \\
\hline 25 & 1221 & 1173 & & 1195 w & 39.47 & 10.96 & 610.76 & 7.58 & 1.60 & 1.40 & $v C_{18} C_{21}(11)+\beta H_{24} C_{21} C_{23}(17)+\beta H_{25} C_{23} N_{19}(30)$ \\
\hline 26 & 1195 & 1148 & $1145 \mathrm{~m}$ & $1149 w$ & 87.67 & 24.34 & 456.90 & 5.67 & 3.39 & 2.85 & $\mathrm{vN}_{11} \mathrm{~N}_{12}(60)$ \\
\hline 27 & 1142 & 1097 & & 1096 w & 146.68 & 40.73 & 527.62 & 6.55 & 2.70 & 2.07 & $v C_{2} C_{9}(10)+v N_{12} C_{14}(40)$ \\
\hline 28 & 1134 & 1090 & & & 69.91 & 19.41 & 242.18 & 3.01 & 1.45 & 1.10 & $\beta \mathrm{H}_{24} \mathrm{C}_{21} \mathrm{C}_{23}(20)+\beta \mathrm{C}_{16} \mathrm{C}_{17} \mathrm{~N}_{19}(12)+\beta \mathrm{H}_{22} \mathrm{C}_{18} \mathrm{C}_{21}(36)$ \\
\hline 29 & 1102 & 1059 & & & 7.47 & 2.07 & 295.96 & 3.67 & 1.26 & 0.90 & $\mathrm{vC}_{4} \mathrm{C}_{5}(15)+\mathrm{BH}_{7} \mathrm{C}_{4} \mathrm{C}_{5}(22)+\mathrm{\beta H}_{6} \mathrm{C}_{5} \mathrm{C}_{4}(47)$ \\
\hline 30 & 1086 & 1044 & & & 5.12 & 1.42 & 134.46 & 1.67 & 3.17 & 2.21 & $v N_{11} N_{12}(24)+v N_{12} C_{14}(16)$ \\
\hline 31 & 1058 & 1017 & 1031 w & $1030 w$ & 1.76 & 0.49 & 393.20 & 4.88 & 3.19 & 2.11 & $\beta C_{16} C_{18} C_{21}(45)$ \\
\hline
\end{tabular}




\begin{tabular}{|c|c|c|c|c|c|c|c|c|c|c|c|}
\hline 32 & 1056 & 1015 & & & 7.59 & 2.11 & 414.19 & 5.14 & 1.76 & 1.15 & $\mathrm{BH}_{7} \mathrm{C}_{4} \mathrm{C}_{5}(14)+\mathrm{\beta H}_{8} \mathrm{C}_{3} \mathrm{C}_{4}(27)+v \mathrm{C}_{3} \mathrm{C}_{4}(35)$ \\
\hline 33 & 1039 & 999 & & & 11.95 & 3.32 & 318.45 & 3.95 & 4.30 & 2.73 & $\beta \mathrm{C}_{17} \mathrm{~N}_{19} \mathrm{C}_{23}(32)+\beta \mathrm{C}_{21} \mathrm{C}_{23} \mathrm{~N}_{19}(15)+\beta \mathrm{C}_{18} \mathrm{C}_{21} \mathrm{C}_{23}(25)$ \\
\hline 34 & 1014 & 974 & & & 2.23 & 0.62 & 6.67 & 0.08 & 1.38 & 0.83 & $\mathrm{TH}_{22} \mathrm{C}_{18} \mathrm{C}_{21} \mathrm{H}_{24}(75)+\Gamma \mathrm{C}_{23} \mathrm{C}_{21} \mathrm{~N}_{19} \mathrm{H}_{25}(18)$ \\
\hline 35 & 987 & 949 & $948 \mathrm{w}$ & $955 \mathrm{w}$ & 0.58 & 0.16 & 8.33 & 0.10 & 1.43 & 0.82 & $\underset{\Gamma C_{23} \mathrm{C}_{21} \mathrm{~N}_{19} \mathrm{H}_{25}(50)+\Gamma \mathrm{C}_{17} \mathrm{C}_{16} \mathrm{~N}_{19} \mathrm{H}_{20}(12)+\Gamma \mathrm{C}_{18} \mathrm{C}_{16} \mathrm{C}_{2}}{\mathrm{H}_{22}(30)}$ \\
\hline 36 & 947 & 910 & & & 1.90 & 0.53 & 6.99 & 0.09 & 1.39 & 0.73 & $\Gamma \mathrm{C}_{17} \mathrm{C}_{16} \mathrm{~N}_{19} \mathrm{H}_{20}(71)$ \\
\hline 37 & 945 & 908 & & $904 w$ & 9.84 & 2.73 & 50.68 & 0.63 & 1.50 & 0.79 & $\mathrm{rH}_{10} \mathrm{C}_{9} \mathrm{~N}_{11} \mathrm{H}_{12}(80)$ \\
\hline 38 & 928 & 891 & $897 \mathrm{w}$ & & 0.39 & 0.11 & 6.15 & 0.08 & 1.38 & 0.70 & $\mathrm{TH}_{6} \mathrm{C}_{5} \mathrm{C}_{4} \mathrm{H}_{7}(46)+\Gamma \mathrm{C}_{3} \mathrm{C}_{2} \mathrm{C}_{4} \mathrm{H}_{8}(32)$ \\
\hline 39 & 921 & 885 & & & 38.10 & 10.58 & 86.38 & 1.07 & 7.12 & 3.56 & $\beta \mathrm{N}_{12} \mathrm{C}_{14} \mathrm{O}_{15}(20)+\beta \mathrm{C}_{14} \mathrm{~N}_{12} \mathrm{~N}_{11}(25)$ \\
\hline 40 & 861 & 827 & $846 \mathrm{w}$ & & 31.22 & 8.67 & 114.39 & 1.42 & 4.31 & 1.88 & $\mathrm{BC}_{4} \mathrm{C}_{5} \mathrm{~S}_{1}(80)$ \\
\hline 41 & 851 & 817 & & & 8.44 & 2.34 & 1.04 & 0.01 & 1.29 & 0.55 & $\mathrm{TH}_{6} \mathrm{C}_{5} \mathrm{C}_{4} \mathrm{H}_{7}(36)+\Gamma \mathrm{C}_{3} \mathrm{C}_{2} \mathrm{C}_{4} \mathrm{H}_{8}(55)$ \\
\hline 42 & 841 & 808 & & & 13.98 & 3.88 & 48.48 & 0.60 & 1.97 & 0.82 & $\begin{array}{c}\mathrm{TH}_{22} \mathrm{C}_{18} \mathrm{C}_{21} \mathrm{H}_{24}(17)+\Gamma \mathrm{C}_{23} \mathrm{C}_{21} \mathrm{~N}_{19} \mathrm{H}_{25}(12)+\Gamma \mathrm{C}_{18} \mathrm{C}_{16} \mathrm{C}_{21} \\
\mathrm{H}_{22}(32)+\Gamma \mathrm{O}_{15} \mathrm{C}_{16} \mathrm{~N}_{12} \mathrm{C}_{14}(12)\end{array}$ \\
\hline 43 & 823 & 791 & & & 25.89 & 7.19 & 107.27 & 1.33 & 4.43 & 1.77 & $v C_{2} C_{3}(10)+\beta C_{2} C_{9} N_{11}(20)+v S_{1} C_{2}(17)$ \\
\hline 44 & 747 & 718 & & & 3.22 & 0.89 & 221.87 & 2.75 & 7.15 & 2.35 & $\mathrm{BC}_{2} \mathrm{C}_{3} \mathrm{C}_{4}(57)+\mathrm{vS} \mathrm{S}_{2}(23)$ \\
\hline 45 & 739 & 710 & $700 w$ & & 31.92 & 8.86 & 64.22 & 0.80 & 2.76 & 0.89 & $\Gamma \mathrm{C}_{18} \mathrm{C}_{16} \mathrm{C}_{21} \mathrm{H}_{22}(25)+\Gamma \mathrm{O}_{15} \mathrm{C}_{16} \mathrm{~N}_{12} \mathrm{C}_{14}(50)$ \\
\hline 46 & 724 & 695 & & & 21.84 & 6.06 & 20.02 & 0.25 & 4.31 & 1.33 & $\begin{array}{c}\beta \mathrm{C}_{21} \mathrm{C}_{23} \mathrm{~N}_{19}(17)+\tau \mathrm{C}_{16} \mathrm{C}_{17} \mathrm{C}_{23} \mathrm{~N}_{19}(16)+\tau \mathrm{C}_{16} \mathrm{C}_{18} \mathrm{C}_{23} \mathrm{C}_{21}( \\
16)+\tau \mathrm{C}_{18} \mathrm{C}_{21} \mathrm{~N}_{19} \mathrm{C}_{23}(10)\end{array}$ \\
\hline 47 & 718 & 690 & & & 16.37 & 4.54 & 7.30 & 0.09 & 3.96 & 1.20 & $\begin{array}{c}\tau C_{16} C_{17} C_{23} N_{19}(27)+\tau C_{16} C_{18} C_{23} C_{21}(22)+\tau C_{18} C_{21} N_{19} \\
C_{23}(16)\end{array}$ \\
\hline 48 & 706 & 679 & & $652 w$ & 75.61 & 20.99 & 10.36 & 0.13 & 1.17 & 0.34 & $\mathrm{\tau H}_{6} \mathrm{C}_{5} \mathrm{C}_{4} \mathrm{H}_{7}(13)+\mathrm{\tau H}_{6} \mathrm{C}_{5} \mathrm{~S}_{1} \mathrm{C}_{2}(81)$ \\
\hline 49 & 633 & 608 & $607 \mathrm{~s}$ & & 4.00 & 1.11 & 49.48 & 0.61 & 7.72 & 1.82 & $\beta C_{16} C_{17} N_{19}(15)+\beta C_{17} N_{19} C_{23}(15)+\beta C_{18} C_{21} C_{23}(41)$ \\
\hline 50 & 610 & 586 & & & 2.58 & 0.72 & 360.42 & 4.47 & 9.05 & 1.98 & $\beta C_{2} S_{1} C_{5}(63)$ \\
\hline 51 & 586 & 563 & & & 1.22 & 0.34 & 16.70 & 0.21 & 3.25 & 0.66 & $\mathrm{TC}_{3} \mathrm{C}_{2} \mathrm{C}_{4} \mathrm{H}_{5}(79)$ \\
\hline 52 & 528 & 507 & $503 \mathrm{~m}$ & & 15.76 & 4.38 & 167.78 & 2.08 & 3.01 & 0.49 & $\begin{array}{c}\mathrm{BC}_{16} \mathrm{C}_{14} \mathrm{~N}_{12}(22)+\tau \mathrm{TH}_{13} \mathrm{~N}_{12} \mathrm{C}_{14} \mathrm{C}_{16}(22)+\Gamma \mathrm{C}_{14} \mathrm{C}_{16} \mathrm{C}_{1}(10) \\
{ }_{11}(10)\end{array}$ \\
\hline 53 & 508 & 488 & & & 44.93 & 12.47 & 72.78 & 0.90 & 1.60 & 0.24 & $\mathrm{TH}_{13} \mathrm{~N}_{12} \mathrm{C}_{14} \mathrm{C}_{16}(61)$ \\
\hline 54 & 492 & 472 & & & 0.31 & 0.09 & 8.91 & 0.11 & 4.01 & 0.57 & $\mathrm{TC}_{2} \mathrm{~S}_{1} \mathrm{C}_{4} \mathrm{C}_{5}(65)$ \\
\hline 55 & 451 & 433 & & & 0.46 & 0.13 & 24.54 & 0.30 & 7.97 & 0.95 & $\begin{array}{c}\beta C_{2} C_{9} N_{11}(14)+v S_{1} C_{2}(12)+\beta C_{17} C_{16} C_{18}(10)+\beta C_{9} C_{2} S_{1} \\
(12)+\beta C_{14} N_{12} N_{11}(11)\end{array}$ \\
\hline 56 & 419 & 402 & & & 7.99 & 2.22 & 9.91 & 0.12 & 4.68 & 0.48 & $\tau \mathrm{CC}_{16} \mathrm{C}_{17} \mathrm{C}_{23} \mathrm{~N}_{19}(15)+\tau \mathrm{C}_{16} \mathrm{C}_{18} \mathrm{C}_{23} \mathrm{C}_{21}(31)$ \\
\hline 57 & 396 & 381 & & & 4.89 & 1.36 & 11.97 & 0.15 & 3.36 & 0.31 & $\begin{array}{c}\tau_{16} C_{18} C_{23} C_{21}(16)+\tau C_{18} C_{21} N_{19} C_{23}(34)+\Gamma C_{14} C_{16} C_{1} \\
{ }_{8} C_{17}(10)\end{array}$ \\
\hline 58 & 379 & 364 & & & 1.32 & 0.37 & 1.86 & 0.02 & 8.03 & 0.68 & $v \mathrm{vC}_{14} \mathrm{C}_{16}(17)+\beta \mathrm{N}_{12} \mathrm{C}_{14} \mathrm{O}_{15}(22)+\beta \mathrm{C}_{17} \mathrm{C}_{16} \mathrm{C}_{18}(20)$ \\
\hline 59 & 340 & 326 & & & 1.88 & 0.52 & 29.62 & 0.37 & 5.99 & 0.41 & $\tau \mathrm{TC}_{2} \mathrm{~S}_{1} \mathrm{C}_{4} \mathrm{C}_{5}(18)+\tau \mathrm{CC}_{2} \mathrm{C}_{9} \mathrm{~N}_{11} \mathrm{~N}_{12}(34)$ \\
\hline 60 & 264 & 253 & & & 13.84 & 3.84 & 34.50 & 0.43 & 4.73 & 0.19 & $\beta C_{14} C_{16} C_{18}(36)$ \\
\hline 61 & 226 & 217 & & $224 \mathrm{w}$ & 11.59 & 3.22 & 71.81 & 0.89 & 3.69 & 0.11 & $\tau C_{5} S_{1} C_{2} C_{9}(11)+\Gamma C_{16} C_{14} N_{12} N_{11}(20)+\tau C_{3} C_{2} C_{9} N_{11}(16)$ \\
\hline 62 & 213 & 205 & & $191 \mathrm{w}$ & 1.15 & 0.32 & 136.24 & 1.69 & 10.18 & 0.27 & $\beta C_{9} C_{2} S_{1}(30)+\beta C_{14} N_{12} N_{11}(22)$ \\
\hline 63 & 170 & 163 & & & 11.98 & 3.33 & 135.90 & 1.69 & 4.69 & 0.08 & $\begin{array}{c}\tau C_{9} \mathrm{~N}_{11} \mathrm{~N}_{12} \mathrm{C}_{14}(20)+\Gamma \mathrm{CC}_{14} \mathrm{C}_{16} \mathrm{C}_{18} \mathrm{C}_{17}(14)+\tau \mathrm{C}_{3} \mathrm{C}_{2} \mathrm{C} \\
{ }_{9} \mathrm{~N}_{11}(13)\end{array}$ \\
\hline 64 & 130 & 125 & & & 1.52 & 0.42 & 83.59 & 1.04 & 5.74 & 0.06 & $\begin{array}{c}\tau \mathrm{CC}_{9} \mathrm{~N}_{11} \mathrm{~N}_{12} \mathrm{C}_{14}(25)+\tau \mathrm{C}_{5} \mathrm{~S}_{1} \mathrm{C}_{2} \mathrm{C}_{9} \\
(37)+\Gamma \mathrm{C}_{14} \mathrm{C}_{16} \mathrm{C}_{18} \mathrm{C}_{17}(10)\end{array}$ \\
\hline 65 & 120 & 115 & & & 6.55 & 1.82 & 60.10 & 0.75 & 5.71 & 0.05 & $\begin{array}{c}\beta C_{2} C_{9} N_{11}(18)+\beta C_{16} C_{14} N_{12}(18)+\beta C_{9} C_{2} S_{1}(17)+\Gamma C_{14} \\
C_{16} C_{18} C_{17}(14)\end{array}$ \\
\hline 66 & 64 & 61 & & $80 \mathrm{~m}$ & 5.89 & 1.63 & 381.03 & 4.73 & 5.90 & 0.01 & $\begin{array}{c}\tau \mathrm{C}_{5} \mathrm{~S}_{1} \mathrm{C}_{2} \mathrm{C}_{9}(12)+\tau \mathrm{C}_{18} \\
\mathrm{C}_{16} \mathrm{C}_{14} \mathrm{~N}_{12}(45)+\tau \mathrm{C}_{3} \mathrm{C}_{2} \mathrm{C}_{9} \mathrm{~N}_{11}(15)\end{array}$ \\
\hline 67 & 46 & 45 & & & 0.73 & 0.20 & 161.56 & 2.01 & 6.68 & 0.01 & $\begin{array}{c}\beta C_{2} C_{9} N_{11}(16)+\beta C_{16} C_{14} N_{12}(14)+\beta C_{9} N_{11} N_{12}(20)+\beta \\
C_{14} N_{12} N_{11}(22)\end{array}$ \\
\hline 68 & 32 & 31 & & & 1.86 & 0.52 & 329.50 & 4.09 & 5.69 & 0.00 & $\begin{array}{c}\tau C_{2} C_{9} N_{11} N_{12}(18)+\tau C_{18} C_{16} C_{14} N_{12}(15)+\tau C_{16} \\
C_{14} N_{12} N_{11}(49)\end{array}$ \\
\hline 69 & 27 & 26 & & & 0.47 & 0.13 & 967.33 & 12.01 & 5.46 & 0.00 & $\begin{array}{c}\tau C_{9} N_{11} N_{12} C_{14}(18)+\tau C_{18} C_{16} C_{14} N_{12}(25)+\tau C_{3} \\
C_{2} C_{9} N_{11}(18)\end{array}$ \\
\hline
\end{tabular}

v: Stretching, $\beta$ : in-plane-bending, $\Gamma$ : out-of-plane bending, $\tau$ - Torsion, vw: very week, w:week, m:medium, s:strong, vs: very strong. ascaling factor: 0.9608 .

belative IR absorption intensities normalized with highest peak absorption equal to 100 .

'Relative Raman intensities calculated by Equation (1) and normalized to 100.

${ }^{\mathrm{d}}$ Total energy distribution calculated at B3LYP/6-311++G(d,p) level. 

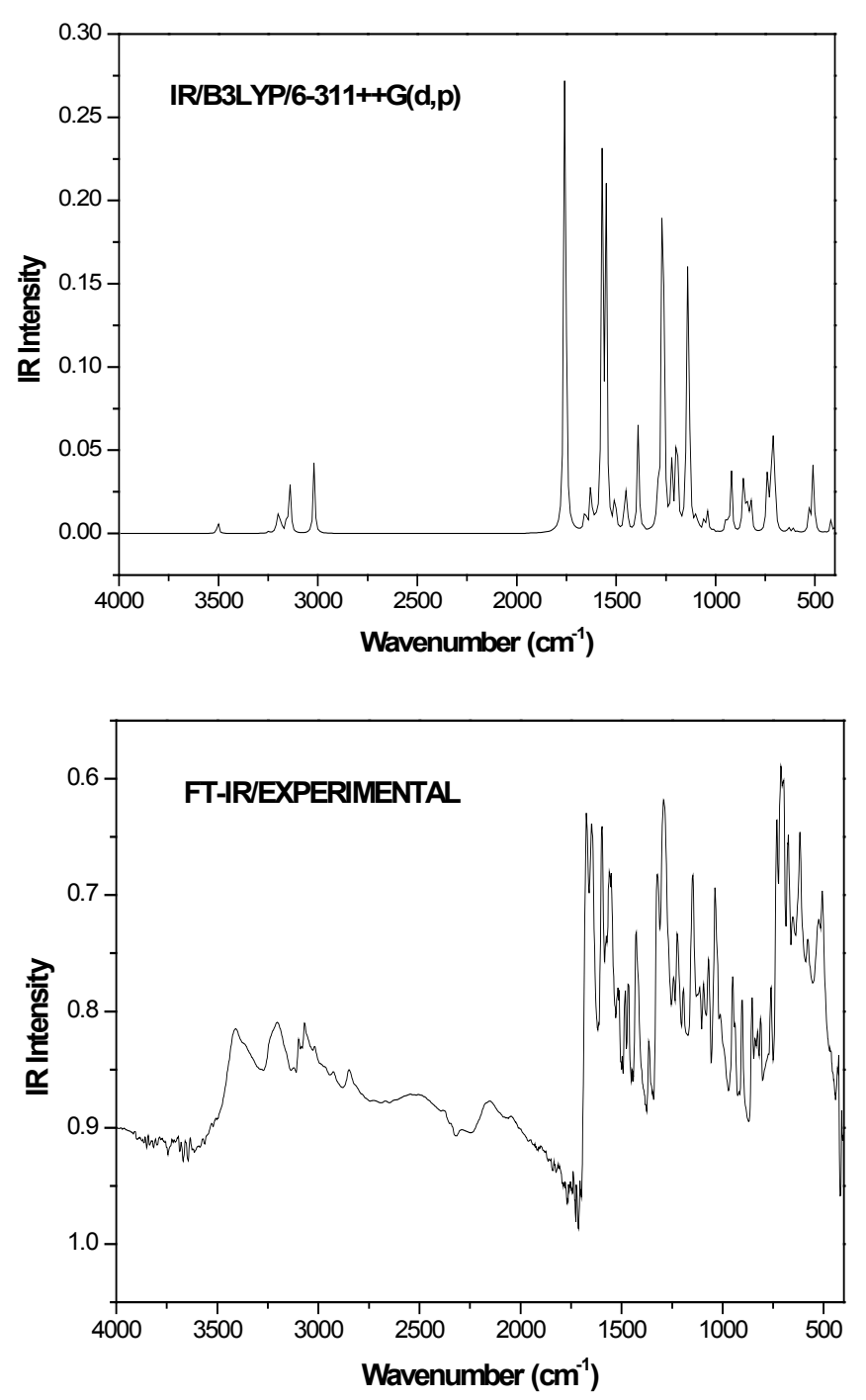

Figure 2 The Theoretical and Experimental FT-IR spectra of $\mathrm{T}_{2} \mathrm{CNH}$.

The $\mathrm{vCH}$ modes are expected to appear in the range of 3100$3000 \mathrm{~cm}^{-1}$ with multiple weak bands and the bands are not affected appreciably by the nature of the substituent's [31,32]. The $\mathrm{VC}-\mathrm{H}$ vibration of the thiophen ring is observed at 3171 $\mathrm{cm}^{-1}$ in FT-IR and $3079 \mathrm{~cm}^{-1}$ in FT-Raman spectra in accordance with the literature [20-22]. The harmonic values corresponding to $\mathrm{vC}-\mathrm{H}$ are calculated in the range $3120-3078 \mathrm{~cm}^{-1}$ (mode nos: $2-4$ ) with TED values $>96 \%$. This indicates that these are highly pure stretching modes. The $\beta \mathrm{C}-\mathrm{H}$ vibrations appear by sharp but weak to medium bands in the range $1100-1500 \mathrm{~cm}^{-1}$ and the bands are not sensitive to the nature of substitutents. The $\mathrm{rC}-\mathrm{H}$ deformation modes are expected to appear in the range $800-1000 \mathrm{~cm}^{-1}$ [32]. The IR active $\beta \mathrm{C}-\mathrm{H} / \Gamma \mathrm{C}-\mathrm{H}$ vibrations of $\mathrm{T} 2 \mathrm{CNH}$ molecule are observed at $1218 / 897 \mathrm{~cm}^{-1}$, respectively. The harmonic wavenumbers of these bands are 1219, 1059, 1015 and $891,817 \mathrm{~cm}^{-1}$ in good agreement with literature values [20-22].

The harmonic frequencies for $\mathrm{VC}-\mathrm{H}, \mathrm{BC}-\mathrm{H}$ and $\mathrm{\Gamma C}-\mathrm{H}$ modes in hydrozone linkage were assigned at 2919, 1315 and $910 \mathrm{~cm}^{-1}$, respectively and these assignments are in line with assignments vC-H: 2901, $\beta C-H:$ 1335, ГC-H: $908 \mathrm{~cm}^{-1}$ (mode nos: 9, 19, 37) are made in the present study. These vibrational assignments are further supported by literature [28] and TED output.

\section{$\mathrm{C}=\mathrm{C}, \mathrm{C}-\mathrm{C}$ vibrations}

In the pyridine ring, the $\mathrm{v}(\mathrm{C}-\mathrm{C})$ stretching vibrations are usually occur in the ranges of $1590-1640,1560-1580$ and $1470-1510 \mathrm{~cm}^{-1}$ [33]. The computed wavenumber for $\mathrm{V}(\mathrm{C}-\mathrm{C})$ modes are lies at 1563, 1542, 1240 and $1173 \mathrm{~cm}^{-1}$ (mode nos: 12, 13, 22 and 25) with TED values. In the present study it has been established well and the FTIR band at $1549 \mathrm{~cm}^{-1}$ and FT-Raman bands at 1241, $1195 \mathrm{~cm}^{-1}$ are designated as $\mathrm{VC}-\mathrm{C}$ vibrations. These assignments are find support from the literature [34] and also from TED values.

The bands arising from BCCC and ГCCC of pyridine moiety are ascribed to bands observed at 1031, 607 and $503 \mathrm{~cm}^{-1}$ respectively in FTIR spectrum. These vibrational assignments find support from harmonic bands: 1017, 999, 608 and 507, 381, 163 $\mathrm{cm}^{-1}$ (mode nos: $31,33,49$ and $52,57,63$ ) in addition to literature values [35].
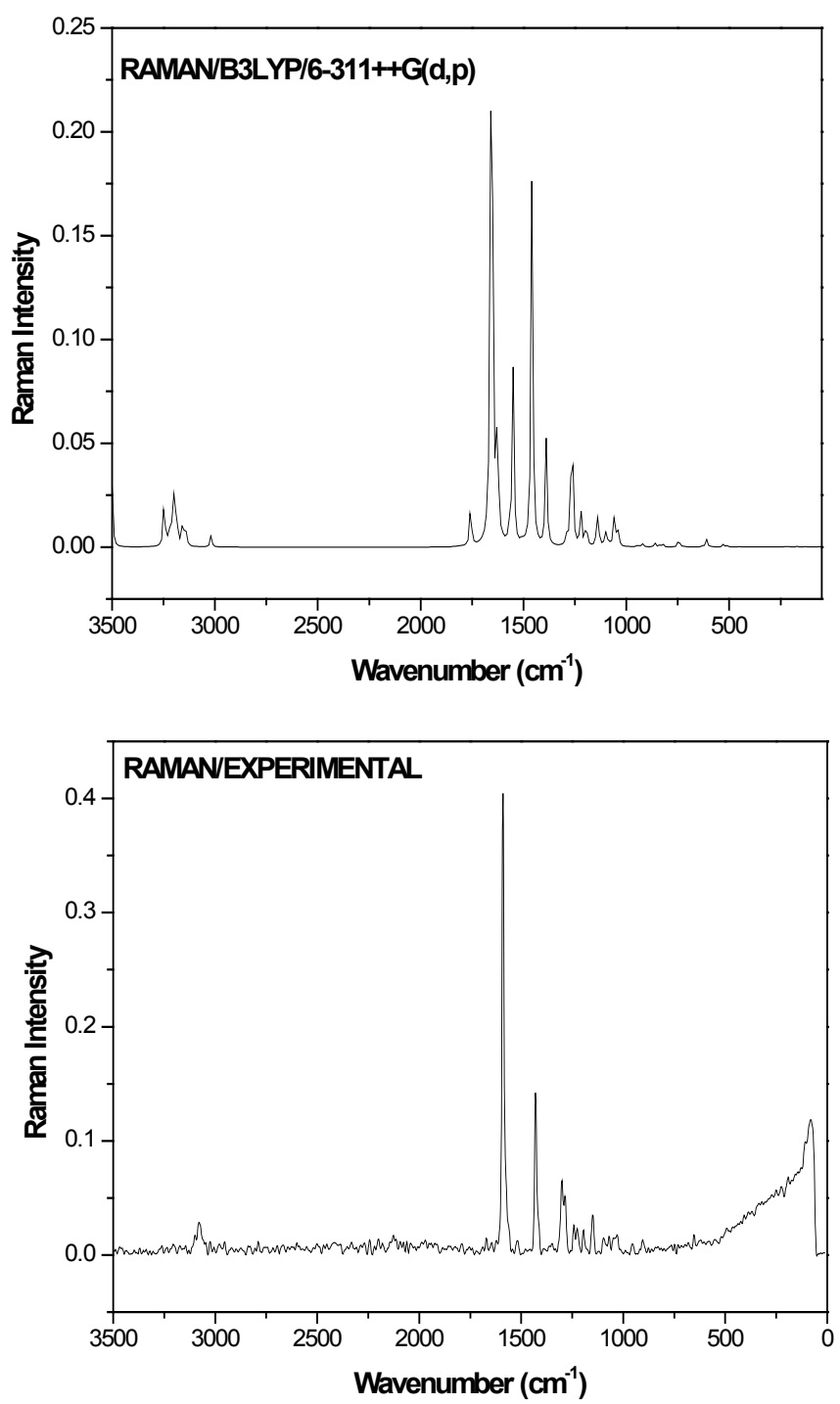

The Theoretical and Experimental FT-Raman spectra of $\mathrm{T}_{2} \mathrm{CNH}$. 


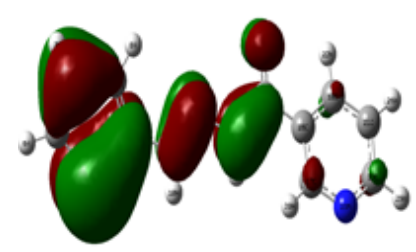

Homo $=-6.248 \mathrm{eV}$

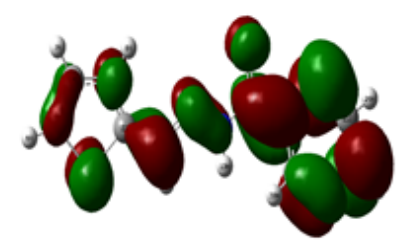

$\mathrm{Lumo}=-2.223 \mathrm{eV}$

Energygap $=4.025 \mathrm{eV}$

Homo-1 $=-7.342 \mathrm{eV}$

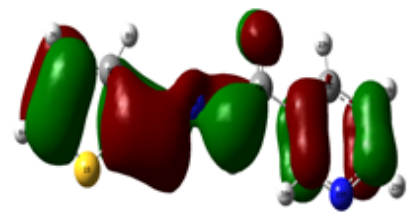

Homo-2 = -7.390 eV

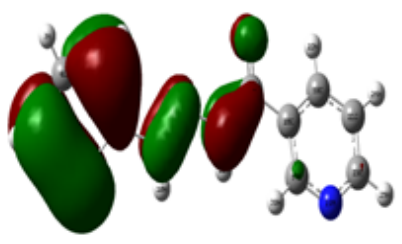

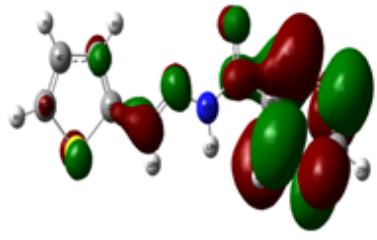

$\mathrm{Lumo}+\mathrm{l}=-1.503 \mathrm{eV}$

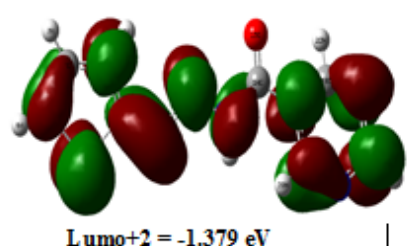

Figure 4 The frontier molecular orbitals for $\mathrm{T}_{2} \mathrm{CNH}$.

The ring carbon-carbon stretching vibrations in thiophen ring are reported in the ranges of 1329-1431, 1420-1501 and 1419-1519 $\mathrm{cm}^{-1}$, respectively [20-22]. For the same mode the computed wavenumbers are: $1508(C=C), 1490(C=C)$ and $1402 \mathrm{~cm}^{-1}(C-C)$. These assignments are having considerable TED values $\geq 15 \%$ and its corresponding mode no: 14 is further supported by observed Raman band $1519 \mathrm{~cm}^{-1}$. The $\beta C C C$ mode of thiophene moiety is ascribed to mode no: $44\left(718 \mathrm{~cm}^{-1}\right)$, this supported by TED value $(57 \%)$ also. The mode nos: 27 and 24 are attributed to $\mathrm{VC}_{2}-\mathrm{C}_{9}$ and $\mathrm{vC}_{14}-\mathrm{C}_{16}$ mode and are in agreement with literature [28].

\section{$\mathrm{C}=\mathrm{N}, \mathrm{C}-\mathrm{N}$ vibrations}

The identifications of $\mathrm{C}=\mathrm{N}$ and $\mathrm{C}-\mathrm{N}$ vibrations is a difficult task, since the mixing of vibrations is possible in this region [36]. However, with the help of Gauss View (3.0) software and TED results, those vibrations are described and assigned in this study. The $\mathrm{C}=\mathrm{N}$ and $\mathrm{C}-\mathrm{N}$ stretching vibrations appear in the ranges of $1670-1600 \mathrm{~cm}^{-1}$ and $1382-1266 \mathrm{~cm}^{-1}$ respectively [36]. In hydrozone linkage, the $v C 9=\mathrm{N} 11$ and $v C 14-\mathrm{N} 12$ stretching vibrations are assigned respectively at $1592 \mathrm{~cm}^{-1}$ (FTIR) /1589 $\mathrm{cm}^{-1}$ (FT-Raman) and at $1096 \mathrm{~cm}^{-1}$ (FT-Raman). The TED results show that these vibrations are mixed with $\mathrm{BHCN}$ and $\mathrm{VC}-\mathrm{C}$ modes and their corresponding harmonic frequencies are 1591 (mode no: 11) and $1097 \mathrm{~cm}^{-1}$ (mode no: 27 ) well correlated with experimental observations. These vibrational assignments are also supported by literature [28] in addition to TED output (75\% and $40 \%)$.
In pyridine ring, the harmonic/observed bands at 1563 (mode no: 12), 1240 (mode no: 22)/1241 $\mathrm{cm}^{-1}$ in FT-Raman spectrum can be assigned to $\mathrm{C}-\mathrm{N}$ stretching vibration and TED also predict that these vibrations are mixed with vC-C modes. These assignments are in good agreement with our earlier study [28] and also find support from TED values (30\%, 43\%). The $\beta C_{21}-C_{23}-N_{19}, \beta C_{16}-C_{17}-N_{19}$ $\beta C_{17}-N_{19}-C_{23}$ and $\beta C_{2}-C_{9}=N_{11}, \beta C_{16}-C_{14}-N_{12}$ deformations belong to pyridine and hydrozone moieties, respectively assigned to 1563 , 1391, 999 and 791, $507 \mathrm{~cm}^{-1}$ (mode nos: 12, 18, 33 and 43, 52). Similarly the $\tau \mathrm{C}_{16} \mathrm{C}_{17} \mathrm{C}_{23} \mathrm{~N}_{19}, \tau \mathrm{C}_{18} \mathrm{C}_{21} \mathrm{~N}_{19} \mathrm{C}_{23}, \tau \mathrm{\tau C}_{3} \mathrm{C}_{2} \mathrm{C}_{9} \mathrm{~N}_{11}, \Gamma \mathrm{C}_{16} \mathrm{C}_{14} \mathrm{~N}_{12} \mathrm{~N}_{11}$ modes are assigned to wavenumbers: $690,381,217,217 \mathrm{~cm}^{-1}$ (mode nos: 47, 57, 61, 61).

\section{$\mathrm{N}-\mathrm{N}$ vibrations}

The vN-N stretching was observed at $1145 \mathrm{~cm}^{-1}$ in FTIR is undoubtedly assigned to $\mathrm{vN}_{11}-\mathrm{N}_{12}$ vibration and the value of this band is calculated at $1148 \mathrm{~cm}^{-1}$ (mode no: 26 ) with a TED of (60\%) [28]. This assignment is well correlated with observed FT-Raman band $1149 \mathrm{~cm}^{-1}$. The in-plane bending vibrations of $\mathrm{C}_{2}-\mathrm{C}_{9}=\mathrm{N}_{11}$, $\mathrm{C}_{9}=\mathrm{N}_{11}-\mathrm{N}_{12} \& \mathrm{C}_{14}-\mathrm{N}_{12}-\mathrm{N}_{11}$ are assigned to wavenumbers: 791, 45 \& 885 , having considerable TED values ( $\geq 20 \%$ ). The wavenumbers $163,326 \mathrm{~cm}^{-1}$ (mode nos: 63, 59) are assigned to $\mathrm{\tau C}_{9}=\mathrm{N}_{11}-\mathrm{N}_{12}-\mathrm{C}_{14^{\prime}}$ $\tau \mathrm{C}_{2}-\mathrm{C}_{9}=\mathrm{N}_{11}-\mathrm{N}_{12}$ modes.

\section{C-S vibrations}

In T2CNH the scaled wavenumbers 791 and $718 \mathrm{~cm}^{-1}$ (mode nos: 43 and 44 ) are assigned to $\mathrm{VC}-\mathrm{S}$ modes of thiophen ring moiety. This assignment is in agreement with the assignments proposed by various authors $[20,21]$. This mode is well known to mix with neighboring modes ( $V C C, \beta C C N /$ mode no: 43 and $\beta C C C /$ mode no: $44)$ as reported in the literature [37]. The harmonic frequencies of $\beta C S C$ and ГCSC vibrations are ascribed to wavenumbers: 586 $\mathrm{cm}^{-1}$ (mode no: 50 ) and $125 \mathrm{~cm}^{-1}$ (mode no: 64 ) with $62 \%$ and $37 \%$ of TED values, respectively. Further the wavenumbers 827 $\mathrm{cm}^{-1}$ (mode no: 40) and $205 \mathrm{~cm}^{-1}$ (mode no: 62) are assigned to $\beta C_{4} C_{5} S_{1}$ and $\beta C_{9} C_{2} S_{1}$ modes, respectively, which are in line with the observed bands ( $846 \mathrm{~cm}^{-1}$ : FTIR and $191 \mathrm{~cm}^{-1}$ : FT-Raman) in addition to support the TED values [80\% and $30 \%$ ].

\section{$\mathrm{C}=\mathrm{O}$ vibrations}

The $\mathrm{C}=\mathrm{O}$ is formed by $\mathrm{P} \pi-\mathrm{P} \pi$ bonding between carbon and oxygen atoms. Carbonyl $(C=O)$ group stretching vibration is expected to appear in the region of $1680-1715 \mathrm{~cm}^{-1}$ [38]. In this study, the carbonyl group stretching vibration appear at $1670 \mathrm{~cm}^{-1}$ as strong band in FT-IR and at $1673 \mathrm{~cm}^{-1}$ as weak band in FT-Raman spectra. The values of $v C=O$ band is calculated at $1688 \mathrm{~cm}^{-1}$ (mode no: 10) with a TED of $85 \%$. The $\beta C=0$ and $\Gamma C=0$ vibrations are assigned respectively to mode nos: 39 and 45 , in which the predicted wavenumber related to $\Gamma \mathrm{C}=\mathrm{O}$ mode is found to be in moderate agreement with the observed FTIR band at $700 \mathrm{~cm}^{-1}$.

\section{NLO Property}

Analysis of organic compounds having conjugated $\pi$-electron systems and large hyperpolarizability using IR and Raman spectroscopy has evolved as a subject of scientific research. The application of the title molecule in the field of non-linear optics demands the investigation of its structural and bonding features 
contributing to the hyperpolarizability enhancement by analyzing the vibrational modes using IR and Raman spectroscopy. The DFT/ B3LYP/6-311++G $(d, p)$ basis set has been used for the prediction of first hyperpolarizability.

The calculated first hyperpolarizability and the total molecular dipole moment of $\mathrm{T} 2 \mathrm{CNH}$ is $1.5861 \times 10^{-30} \mathrm{esu}, 0.9906$ Debye, respectively obtained by $B 3 L Y P / 6-311++G(d, p)$ level of theory. The total dipole moment of the title molecule is moderately equal and $\beta O$ value of $\mathrm{T} 2 \mathrm{CNH}$ is 4 times greater than that of urea, hence the molecule $\mathrm{T} 2 \mathrm{CNH}$ has considerable non-linear optical (NLO) activity and the hyperpolarizabilities of T2CNH are given in Table 3.

\section{NBO Analysis}

This method gives information about the intra- and inter-molecular interactions among bonds. Furthermore, it provides a convenient basis for investigating the interactions in both filled and virtual orbital spaces along with charge transfer and conjugative interactions in molecular system [39]. The natural bonding orbital (NBO) analysis was performed for T2CNH using B3LYP/6$311++\mathrm{G}(\mathrm{d}, \mathrm{p})$ basis set in order to elucidate the intra-molecular, hybridization and delocalization of ED within $\mathrm{T} 2 \mathrm{CNH}$. The strong intra-molecular hyperconjugative interaction of the $\sigma$ and $\pi$ electrons of $\mathrm{C}-\mathrm{C}$ to the anti $\mathrm{C}-\mathrm{C}$ bond of the pyridine ring leads to stabilization of some part of the pyridine ring. The NBO analysis has been carried out by B3LYP/6-311++G(d,p) basis set and the $E(2)$ values and types of the transition are shown in Table 4.

Table 3 The NLO measurements of $\mathrm{T}_{2} \mathrm{CNH}$.

\begin{tabular}{|c|c|}
\hline Parameters & $B 3 L Y P / 6-311++G(d, p)$ \\
\hline Dipole moment ( $\mu$ ) & Debye \\
\hline$\mu_{x}$ & 0.3145 \\
\hline$\mu_{v}$ & -0.7701 \\
\hline$\mu_{z}$ & 0.5380 \\
\hline$\mu$ & 0.9906Debye \\
\hline Polarizability $\left(\alpha_{0}\right)$ & $\times 10^{-30}$ esu \\
\hline$\alpha_{x x}$ & 330.88 \\
\hline$\alpha_{x y}$ & -3.11 \\
\hline$\alpha_{y y}$ & 168.52 \\
\hline$\alpha_{x z}$ & -4.63 \\
\hline$\alpha_{y z}$ & -0.11 \\
\hline$\alpha_{2 z}$ & 97.39 \\
\hline$\alpha_{0}$ & $0.5318 \times 10^{-30} \mathrm{esu}$ \\
\hline Hyperpolarizability $\left(\beta_{0}\right)$ & $\times 10^{-30}$ esu \\
\hline$\beta_{x x x}$ & -1853.85 \\
\hline$\beta_{x x y}$ & 229.60 \\
\hline$\beta_{x y y}$ & -33.45 \\
\hline$\beta_{y y y}$ & -129.96 \\
\hline$\beta_{x x z}$ & 40.31 \\
\hline$\beta_{x y z}$ & 21.11 \\
\hline$\beta_{y y z}$ & 2.92 \\
\hline$\beta_{x z z}$ & 54.46 \\
\hline$\beta_{y z z}$ & -29.90 \\
\hline$\beta_{z z z}$ & -38.55 \\
\hline$\beta_{0}$ & $1.5861 \times 10^{-30} \mathrm{esu}$ \\
\hline
\end{tabular}

Standard value for urea $\left(\boldsymbol{\mu}=1.3732\right.$ Debye, $\boldsymbol{\beta}_{0}=0.3728 \times 10^{-30} \mathrm{esu}$ ): esuelectrostatic unit
The larger $E(2)$ value shows the intensive interaction between electron donors and electron acceptors. The strong intramolecular hyper conjugative interactions of the $\sigma$ and $\pi$ electrons of the $\mathrm{C}=\mathrm{C}, \mathrm{C}=\mathrm{N}$ to the anti $\mathrm{C}=\mathrm{C}, \mathrm{C}=\mathrm{N}$ bond of the ring as well as $\mathrm{C}=\mathrm{O}$ group leads to stabilization of some part of the ring system in $\mathrm{T}_{2} \mathrm{CNH}$. In the present study, the $\pi$-character of the bond plays an important role on comparing with $\sigma$ bond character. The hyper conjugative interactions $\pi\left(C_{2}-C_{3}\right) \rightarrow \pi^{*}\left(C_{9}-N_{11}\right), \pi\left(C_{4}-\right.$ $\left.\mathrm{C}_{5}\right) \rightarrow \pi^{*}\left(\mathrm{C}_{2}-\mathrm{C}_{3}\right), \quad \pi\left(\mathrm{C}_{16}-\mathrm{C}_{17}\right) \rightarrow \pi^{*}\left(\mathrm{C}_{18}-\mathrm{C}_{21}\right), \quad \pi\left(\mathrm{C}_{18}-\mathrm{C}_{21}\right) \rightarrow \pi^{*}\left(\mathrm{~N}_{19}-\mathrm{C}_{23}\right)$, $\pi\left(\mathrm{N}_{19}-\mathrm{C}_{23}\right) \rightarrow \pi^{*}\left(\mathrm{C}_{16}-\mathrm{C}_{17}\right)$ and $\pi\left(\mathrm{C}_{14}-\mathrm{O}_{15}\right) \rightarrow \pi^{*}\left(\mathrm{C}_{16}-\mathrm{C}_{17}\right)$ transfer stabilization energy: $76.65,66.02,89.54,122.13,112.97$ and $15.10 \mathrm{KJ} / \mathrm{mol}$ to the molecular system. The lone pair of sulphar, nitrogen and oxygen atoms play great role in $\mathrm{T}_{2} \mathrm{CNH}$ molecule. The $\mathrm{S}_{1}, \mathrm{~N}_{12}$ \& $\mathrm{O}_{15}$ atoms transfer maximum energy 91.55, 190.41 and $118.20 \mathrm{KJ} / \mathrm{mol}$ to $\left(\mathrm{C}_{4}-\mathrm{C}_{5}\right),\left(\mathrm{C}_{14}-\mathrm{O}_{15}\right) \&\left(\mathrm{~N}_{12}-\mathrm{C}_{14}\right)$ bonds, respectively. The maximum hyperconjucative $E(2)$ energy of heteroatoms during the inter-molecular interaction leads the molecule towards medicinal and biological applications [28]. The bond $\sigma\left(\mathrm{C}_{3}-\mathrm{H}_{8}\right)$ transfer more energy $(23.39 \mathrm{KJ} / \mathrm{mol})$ to $\sigma^{*}\left(\mathrm{~S}_{1}-\mathrm{C}_{2}\right)$ bond on comparing with energy transfer $(18.95 \mathrm{KJ} / \mathrm{mol})$ from $\sigma\left(\mathrm{C}_{4}-\mathrm{H}_{7}\right)$ to $\sigma^{*}\left(\mathrm{~S}_{1}-\mathrm{C}_{5}\right)$. Hence the $v\left(\mathrm{~S}_{1}-\mathrm{C}_{2}\right)$ mode observe at higher frequency $791 \mathrm{~cm}^{-1}$ (mode no: 43 ) than the $v\left(\mathrm{~S}_{1}-\mathrm{C}_{5}\right)$ mode $(718$ $\mathrm{cm}^{-1} /$ mode no: 44$)$.

\section{HOMO-LUMO Analysis}

The highest occupied molecular orbital (HOMO) and lowest unoccupied molecular orbital (LUMO) analysis is very important parameters for quantum chemistry. The energy values of HOMO ( $\pi$-donor) and LUMO ( $\pi$-acceptor) and its energy gap which reflects the chemical activity of the molecule. The HOMO and LUMO energy was calculated by B3LYP/6-311++G(d,p) level of theory. The frontier molecular orbitals (FMOs) of $\mathrm{T} 2 \mathrm{CNH}$ are listed in Table 5. The atomic compositions of FMOs are shown in Figure 4. The HOMO is located over the thiophene and hydrozone moieties. The LUMO is located over pyridine ring. The LUMO transition implies that an ED transfer to pyridine ring via hydrazone linkage. The HOMO and LUMO energies are predicted as $-6.248 \mathrm{eV}$ and $-2.223 \mathrm{eV}$, respectively. The calculated HOMOLUMO energy gap is $4.025 \mathrm{eV}$, which explains the eventual charge transfer taking place within the present molecule. The physicochemical properties are also listed in Table 6.

\section{UV-Vis Spectral Analysis}

The UV-Visible absorption spectrum of $\mathrm{T} 2 \mathrm{CNH}$ was recorded in the range of $250-350 \mathrm{~nm}$ is shown in Figure 5. All the structures allow strong $\pi-\pi^{*}$ (or) $\sigma-\sigma^{*}$ transition in the UV-Vis region with high extinction coefficients. On the basis of fully optimized ground state structure at TD-DFT/B3LYP/6-311++G(d,p) calculation has been used to determine the low-lying excited states of $\mathrm{T}_{2} \mathrm{CNH}$. The calculated results involving the vertical excitation energies, oscillator strength ( $f$ ) and wavelength are carried out and compared with measured experimental wavelength. Typically, according to Franck-Condon principle, the maximum absorption peaks ( $\lambda$ max) in a UV-Vis spectrum corresponds to vertical excitation. It is evident from the Table 7 that the calculated absorption maxima values have been found to be 332, 301 and 
Table 4 The second order perturbation theory analysis of Fock Matrix in NBO basis for T2CNH.

\begin{tabular}{|c|c|c|c|c|c|c|c|}
\hline Type & Donor NBO (i) & ED/e & Acceptor NBO (j) & ED/e & $\mathrm{E}^{(2)} \mathrm{kJ} / \mathrm{mol}$ & $E(j)-E(i)$ a.u. & $F(i, j)$ a.u. \\
\hline \multirow[t]{2}{*}{$\pi-\pi^{*}$} & $\mathrm{BD}(2) \mathrm{C} 2-\mathrm{C} 3$ & 1.795 & $B D *(2) C 4-C 5$ & 0.309 & 69.58 & 0.28 & 0.06 \\
\hline & & & $B D^{*}(2) C 9-N 11$ & 0.209 & 76.65 & 0.28 & 0.06 \\
\hline \multirow[t]{3}{*}{$\sigma-\sigma^{*}$} & $\mathrm{BD}(1) \mathrm{C} 3-\mathrm{H} 8$ & 1.972 & $B D *(1) S 1-C 2$ & 0.028 & 23.39 & 0.73 & 0.06 \\
\hline & & & $B D *(1) C 2-C 3$ & 0.021 & 7.57 & 1.12 & 0.04 \\
\hline & & & $B D^{*}(1) C 4-C 5$ & 0.014 & 8.08 & 1.11 & 0.04 \\
\hline$\pi-\pi^{*}$ & $\mathrm{BD}(2) \mathrm{C} 4-\mathrm{C} 5$ & 1.846 & $B D^{*}(2) C 2-C 3$ & 0.351 & 66.02 & 0.30 & 0.06 \\
\hline \multirow[t]{3}{*}{$\sigma-\sigma^{*}$} & $\mathrm{BD}(1) \mathrm{C} 4-\mathrm{H} 7$ & 1.976 & $B D *(1) S 1-C 5$ & 0.017 & 18.95 & 0.75 & 0.05 \\
\hline & & & $B D *(1) C 2-C 3$ & 0.021 & 8.03 & 1.12 & 0.04 \\
\hline & & & $B D *(1) C 4-C 5$ & 0.014 & 5.73 & 1.12 & 0.04 \\
\hline$\pi-\pi^{*}$ & $\mathrm{BD}(2) \mathrm{C} 14-\mathrm{O} 15$ & 1.980 & $B D *(2) \mathrm{C} 16-\mathrm{C} 17$ & 0.335 & 15.10 & 0.40 & 0.04 \\
\hline \multirow[t]{3}{*}{$\pi-\pi^{*}$} & BD (2) C16 - C17 & 1.633 & $B D *(2) \mathrm{C} 14-015$ & 0.277 & 65.98 & 0.3 & 0.06 \\
\hline & & & $B D *(2) C 18-C 21$ & 0.277 & 89.54 & 0.29 & 0.07 \\
\hline & & & $B D *(2) N 19-C 23$ & 0.366 & 69.71 & 0.27 & 0.06 \\
\hline \multirow[t]{2}{*}{$\pi-\pi^{*}$} & $\mathrm{BD}(2) \mathrm{C} 18$ - C21 & 1.636 & $B D *(2) \mathrm{C} 16-\mathrm{C} 17$ & 0.335 & 74.85 & 0.28 & 0.06 \\
\hline & & & $B D *(2) N 19-C 23$ & 0.366 & 122.13 & 0.27 & 0.08 \\
\hline \multirow[t]{2}{*}{$\pi-\pi^{*}$} & BD (2) N19 - C23 & 1.706 & $\mathrm{BD} *(2) \mathrm{C} 16-\mathrm{C} 17$ & 0.335 & 112.97 & 0.32 & 0.08 \\
\hline & & & $B D *(2) \mathrm{C} 18-\mathrm{C} 21$ & 0.277 & 52.59 & 0.33 & 0.06 \\
\hline \multirow[t]{2}{*}{$n-\pi^{*}$} & LP (2) S1 & 1.622 & $B D *(2) C 2-C 3$ & 0.351 & 87.15 & 0.27 & 0.07 \\
\hline & & & $B D *(2) C 4-C 5$ & 0.309 & 91.55 & 0.26 & 0.07 \\
\hline \multirow[t]{3}{*}{$n-\pi^{*}$} & LP (2) N12 & 1.667 & BD*(2) C9 - N11 & 0.209 & 115.14 & 0.29 & 0.08 \\
\hline & & & $B D *(2) \mathrm{C} 14-015$ & 0.017 & 4.44 & 0.88 & 0.03 \\
\hline & & & $B D *(2) C 14-015$ & 0.277 & 190.41 & 0.32 & 0.11 \\
\hline \multirow[t]{2}{*}{$n-\sigma^{*}$} & LP (1) 015 & 1.855 & BD*(1) N12 - C14 & 0.084 & 118.2 & 0.67 & 0.12 \\
\hline & & & BD*(1) C14 - C16 & 0.069 & 80.37 & 0.66 & 0.10 \\
\hline$n-\sigma^{*}$ & LP (1) N19 & 1.916 & $\mathrm{BD}^{*}(1) \mathrm{C} 16-\mathrm{C} 17$ & 0.033 & 39.33 & 0.90 & 0.08 \\
\hline
\end{tabular}

Table 5 The frontier molecular orbital of $\mathrm{T}_{2} \mathrm{CNH}$.

\begin{tabular}{|c|c|c|c|}
\hline Occupancy & Orbital energies (a.u) & Orbital energies (eV) & Kinetic energies (a.u) \\
\hline $\mathrm{O}_{56}$ & -0.292 & -7.947 & 1.641 \\
\hline $\mathrm{O}_{57}$ & -0.284 & -7.740 & 1.641 \\
\hline $\mathrm{O}_{58}$ & -0.271 & -7.390 & 1.582 \\
\hline $\mathrm{O}_{59}$ & -0.269 & -7.342 & 2.186 \\
\hline $\mathrm{O}_{60}$ & -0.229 & -6.248 & 1.567 \\
\hline $\mathrm{V}_{61}$ & -0.081 & -2.223 & 1.590 \\
\hline $\mathrm{V}_{62}$ & -0.055 & -1.503 & 1.586 \\
\hline $\mathrm{V}_{63}$ & -0.055 & -1.503 & 1.424 \\
\hline $\mathrm{V}_{64}$ & -0.019 & -0.519 & 0.488 \\
\hline $\mathrm{V}_{65}$ & -0.009 & -0.255 & 0.535 \\
\hline
\end{tabular}

Table 6 The Physico-Chemical properties of $\mathrm{T}_{2} \mathrm{CNH}$.

\begin{tabular}{|c|c|}
\hline Parameters & Values \\
\hline HOMO & $-6.248 \mathrm{eV}$ \\
\hline LUMO & $-2.223 \mathrm{eV}$ \\
\hline Energy gap & $4.025 \mathrm{eV}$ \\
\hline Ionization potential (IP) & $6.248 \mathrm{eV}$ \\
\hline Electron affinity (EA) & $2.223 \mathrm{eV}$ \\
\hline Electrophilicity Index $(\omega)$ & 2.562 \\
\hline Chemical Potential $(\mu)$ & 7.359 \\
\hline Electro negativity $(\chi)$ & -7.359 \\
\hline Hardness $(\eta)$ & -4.025 \\
\hline
\end{tabular}

$286 \mathrm{~nm}$ which correlates well with the experimental values 340 and $290 \mathrm{~nm}$. The more intense band at $340 \mathrm{~nm}$ has maximum oscillator strength $(f=0.6909)$, corresponds to Homo-Lumo transition and is mostly characterized as $n-\pi^{*}$ type. This type of transition is attributed to the presence of large no of free lone pairs of electrons available on sulphar $\left(S_{1}\right)$, Nitrogen $\left(N_{11}, N_{12}\right)$ and oxygen $\left(\mathrm{O}_{15}\right)$ atoms. The experimental and theoretical UVVis absorption spectrum is shown in Figure 5. The density of states spectrum of $\mathrm{T} 2 \mathrm{CNH}$ is shown in Figure 6 . It was used to calculate group contributions to the molecular orbitals (HOMO and LUMO). DOS plot shows population analysis per orbital and demonstrates a simple view of the character of the molecular orbitals (MOs) in a certain energy range. 

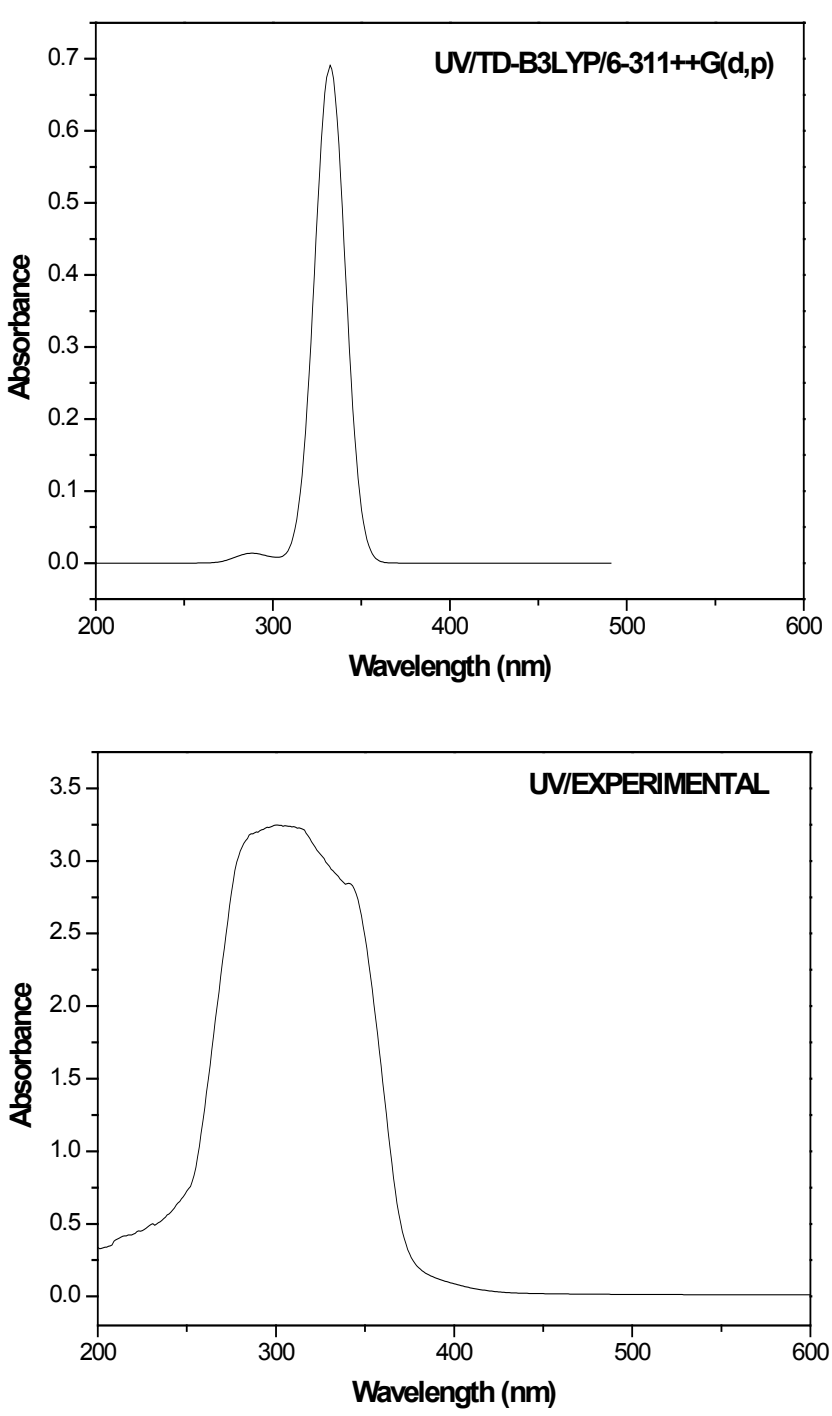

Figure 5 The Theoretical and Experimental UV-Visible spectra of $\mathrm{T}_{2} \mathrm{CNH}$.

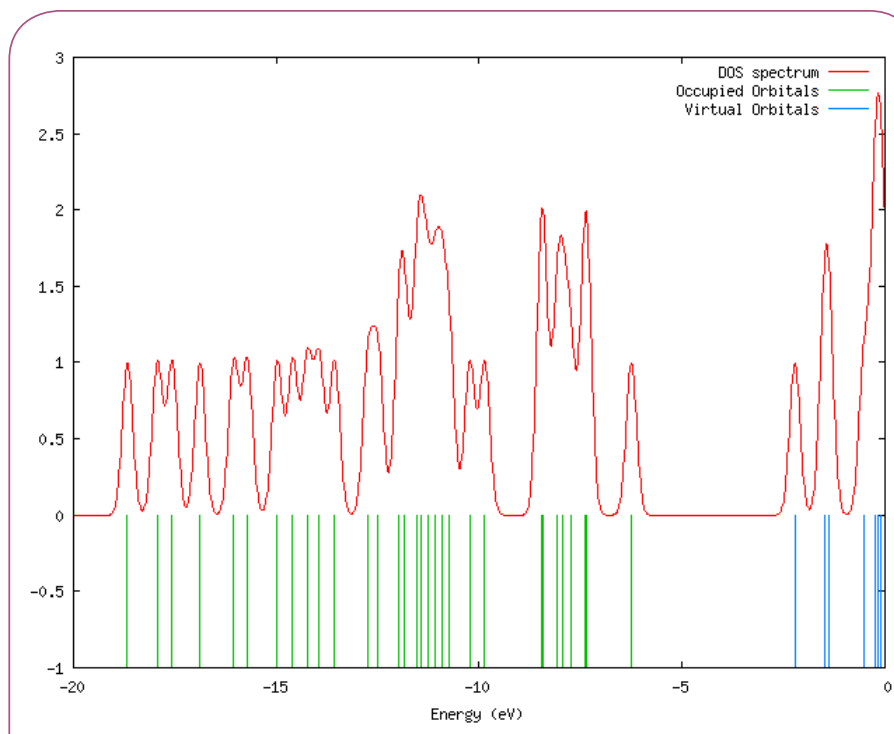

Figure 6 The DOS spectrum of $\mathrm{T}_{2} \mathrm{CNH}$.

\section{MEP Analysis}

The molecular electrostatic potential (MEP) is widely used as a reactivity map displaying most probable regions for the electrophilic attack of charged part on organic molecule. MEP plot provides a simple way in predicting the interaction of different geometries. In order to predict the reactive sites for electrophilic and nucleophilic attacks of the $\mathrm{T}_{2} \mathrm{CNH}, \mathrm{MEP}$ was calculated with DFT/B3LYP/6-311++G(d,p) level of theory. The negative (red color) and positive (blue color) regions of MEP are related to electrophilic and nucleophilic reactivity respectively is shown in Figure 7. The negative region is located over the carbonyl group and the positive region is located over Hydrogen atom in the hydrazone linkage.

\section{Mulliken Charges Analysis}

The Mulliken atomic charge calculation has an important role in the application of quantum chemical calculation to molecular system, because the atomic charges should affect dipole moment, polarizability, electronic structure and more a lot of properties of molecular systems. The total atomic charges of $\mathrm{T} 2 \mathrm{CNH}$ are calculated by Mulliken population analysis with B3LYP/6-311++G $(d, p)$ basis set and its values are listed in Table 8. The Mulliken atomic charge plot for T2CNH is shown in Figure 8. The $C_{9} / C_{3}$

Table 7 The electronic transition of $\mathrm{T} 2 \mathrm{CNH}$.

\begin{tabular}{|c|c|c|c|c|}
\hline $\begin{array}{l}\text { Calculated at } \\
\text { B3LYP/ } \\
6-311++G(d, p)\end{array}$ & $\begin{array}{l}\text { Oscillator } \\
\text { strength }\end{array}$ & $\begin{array}{l}\text { Calculated } \\
\text { Band gap } \\
\text { (ev/nm) }\end{array}$ & $\begin{array}{l}\text { Experimental } \\
\text { Band gap } \\
\text { (nm) }\end{array}$ & Type \\
\hline Excited State 1 & $\begin{array}{c}\text { Singlet-A } \\
(\mathrm{f}=0.6909)\end{array}$ & $\begin{array}{c}3.7311 \\
\mathrm{eV} / 332.30 \mathrm{~nm}\end{array}$ & 340 & $\pi-\pi^{*}$ \\
\hline $60->61$ & 0.6388 & -4.0254 & & \\
\hline $60->62$ & 0.1325 & -4.7451 & & \\
\hline Excited State 2 & $\begin{array}{c}\text { Singlet-A } \\
(\mathrm{f}=0.0043)\end{array}$ & $\begin{array}{c}4.1118 \\
\mathrm{eV} / 301.54 \mathrm{~nm}\end{array}$ & & \\
\hline $59->61$ & 0.6467 & -5.1187 & & \\
\hline $59->62$ & 0.1819 & -5.8384 & & \\
\hline Excited State 3 & $\begin{array}{c}\text { Singlet-A } \\
(f=0.0126)\end{array}$ & $\begin{array}{c}4.3234 \\
\mathrm{eV} / 286.77 \mathrm{~nm}\end{array}$ & 290 & $\pi-\pi^{*}$ \\
\hline $60->62$ & 0.4175 & -4.7451 & & \\
\hline $60->63$ & 0.5580 & -4.8692 & & \\
\hline
\end{tabular}

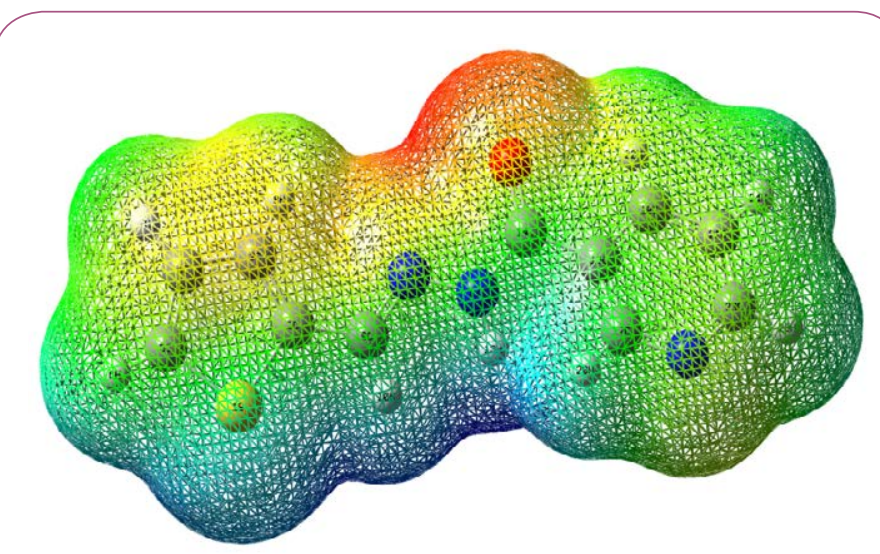

Figure 7 Molecular electrostatic potential map of $\mathrm{T}_{2} \mathrm{CNH}$. 
atoms have the highest negative/positive charges, respectively among the other atoms in $\mathrm{T} 2 \mathrm{CNH}$ due to the resonance.

\section{Thermodynamical Properties}

Several thermodynamic parameters have been calculated using DFT/B3LYP/ 6-311++G(d,p) basis set are listed in Table 9. The thermodynamic parameters viz, entropy $\left(\mathrm{S}^{0}{ }_{m}\right)$, enthalpy $\left(\Delta \mathrm{H}^{0}{ }_{m}\right)$ and heat capacity at constant pressure $\left(\mathrm{C}_{p, m}^{0}\right)$ for $\mathrm{T} 2 \mathrm{CNH}$ was calculated from the theoretical harmonic wavenumbers obtained from $B 3 L Y P / 6-311++G(d, p)$ basis set in the range $100-1000 k$ and listed in Table 10. It is evident from the Table $\mathbf{1 0}$ that the thermodynamic parameters increase with rise of temperature

Table 8 The Mulliken atomic charges of $\mathrm{T}_{2} \mathrm{CNH}$.

\begin{tabular}{|c|c|c|c|c|c|}
\hline Atoms & Charges & Atoms & Charges & Atoms & Charges \\
\hline S1 & -0.6516 & H10 & 0.1263 & N19 & -0.0124 \\
\hline C2 & 0.2759 & N11 & 0.1794 & H20 & 0.1758 \\
\hline C3 & 1.9917 & N12 & -0.0550 & C21 & -0.0964 \\
\hline C4 & -1.2036 & H13 & 0.2477 & H22 & 0.2280 \\
\hline C5 & 0.4451 & C14 & -0.7857 & C23 & -0.3517 \\
\hline H7 & 0.2687 & O15 & -0.2925 & H24 & 0.1897 \\
\hline H8 & 0.1582 & C16 & 0.9920 & H25 & 0.1903 \\
\hline C9 & -1.7752 & C17 & -0.5899 & & \\
\hline
\end{tabular}

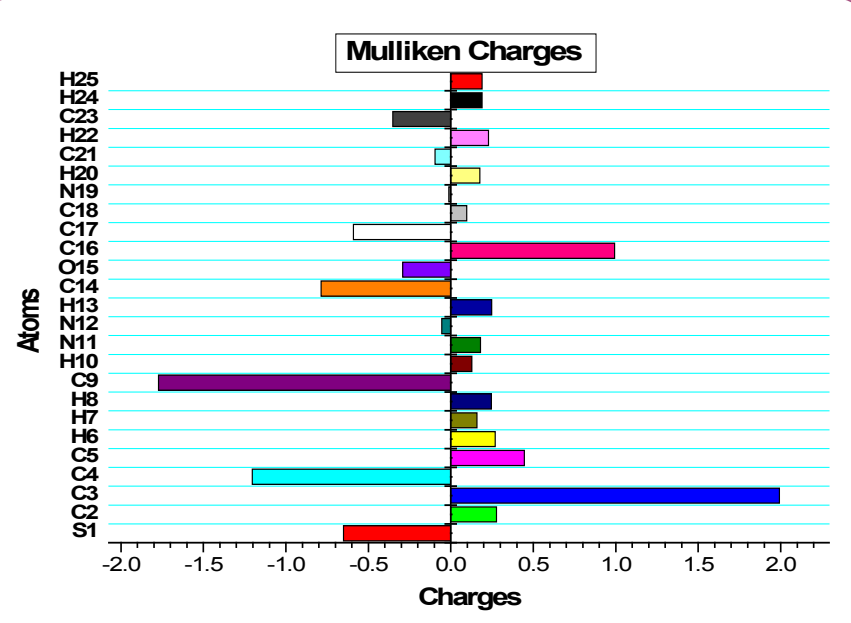

Figure 8 The Mulliken atomic charges of $\mathrm{T}_{2} \mathrm{CNH}$.

Table 9 The calculated total energy (a.u), zero point vibrational energies $(\mathrm{kcal} / \mathrm{mol})$, rotational constants $(\mathrm{GHz})$ and entropy $\left(\mathrm{cal} / \mathrm{mol} \mathrm{K}^{-1}\right)$ for $\mathrm{T}_{2} \mathrm{CNH}$.

\begin{tabular}{|c|c|}
\hline Parameters & B3LYP/6-311++G(d,p) \\
\hline Total Energies & -1062.41677 \\
\hline Zero-point Energy & $114.99338($ Kcal/Mol) \\
\hline Rotational constants (GHZ) & 1.78813 \\
\hline & 0.15572 \\
\hline & 0.14465 \\
\hline Entropy & 122.336 \\
\hline Total & 42.214 \\
\hline Translational & 33.345 \\
\hline Rotational & 46.777 \\
\hline Vibrational & \\
\hline
\end{tabular}

due to the fact that the molecular vibrational intensities increase with temperature. The correlation equations between these thermodynamic parameters and temperature were fitted by parabolic formula and the regression coefficient is also given in the parabolic equation. The correlation relation between the thermodynamic parameters and temperature are as follows:

$\mathrm{C}^{0}{ }_{\mathrm{p}, \mathrm{m}}=4.44266+0.01876 \mathrm{~T}+1.6571 \times 10^{-5} \mathrm{~T}^{2}\left(\mathrm{R}^{2}=0.99929\right)$

$\mathrm{S}_{\mathrm{m}}^{0}=1.35549+0.00572 \mathrm{~T}+5.0560 \times 10^{-5} \mathrm{~T}^{2}\left(\mathrm{R}^{2}=0.99997\right)$

$\Delta \mathrm{H}_{\mathrm{m}}^{0}=3.15466+0.01332 \mathrm{~T}+1.1767 \times 10^{-5} \mathrm{~T}^{2}\left(\mathrm{R}^{2}=0.99944\right)$

The correlation graphs between various thermodynamic functions and temperature are graphically presented in Figure 9.

\section{Conclusion}

The complete vibrational analysis has been performed to using quantum chemical calculation at DFT method for the first time to the title molecule $\mathrm{T}_{2} \mathrm{CNH}$. The calculated bond parameters are good agreement with the related single crystal $\mathrm{X}$-ray diffraction (XRD) data. In $\mathrm{T}_{2} \mathrm{CNH}$, the thiophene and hydrazone moieties are co-planar, which shows a good conjuction between p-orbitals of all atoms of thiophene and hydrazone moieties. The vibrational

Table 10 Thermodynamic properties of $\mathrm{T} 2 \mathrm{CNH}$ at different temperatures.

\begin{tabular}{|c|c|c|c|}
\hline $\mathrm{T}(\mathrm{K})$ & $\mathrm{S}\left(\mathrm{Jmol}^{-1} \mathrm{~K}^{-1}\right)$ & $\mathrm{Cp}\left(\mathrm{Jmol}^{-1} \mathrm{~K}^{-1}\right)$ & $\mathrm{ddH}\left(\mathrm{kJmol}^{-1}\right)$ \\
\hline 100 & 349.07 & 100 & 6.95 \\
\hline 200 & 436.15 & 159.71 & 19.86 \\
\hline 298.15 & 511.96 & 224.53 & 38.7 \\
\hline 300 & 513.36 & 225.74 & 39.12 \\
\hline 400 & 586.95 & 287.34 & 64.85 \\
\hline 500 & 656.77 & 338.54 & 96.24 \\
\hline 600 & 722.24 & 379.34 & 132.21 \\
\hline 700 & 783.25 & 411.8 & 171.83 \\
\hline 800 & 840.01 & 438.01 & 214.36 \\
\hline 900 & 892.88 & 459.52 & 259.27 \\
\hline 1000 & 942.25 & 477.43 & 306.15 \\
\hline
\end{tabular}

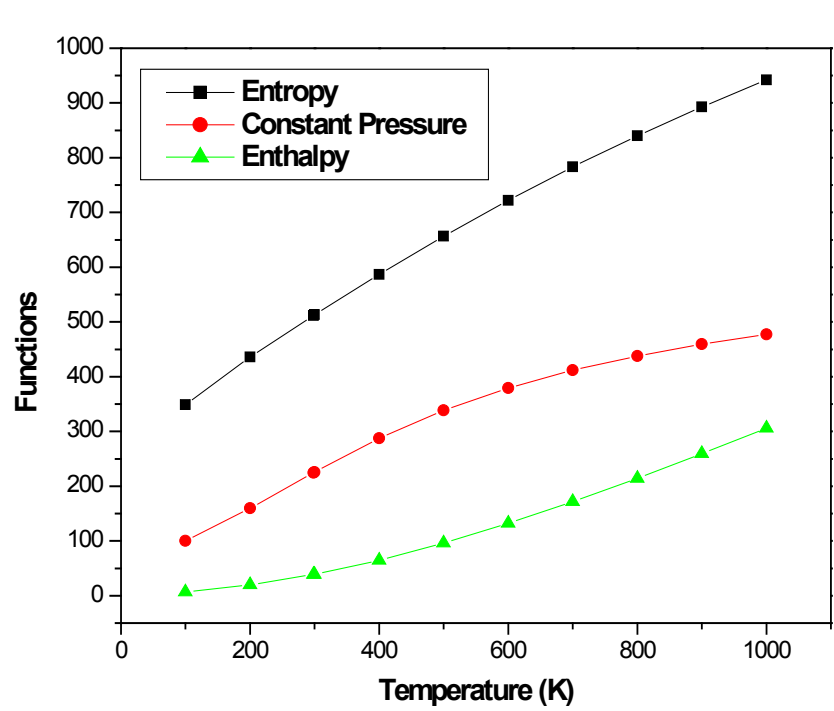

Figure 9

The thermodynamic properties of $\mathrm{T} 2 \mathrm{CNH}$ at different temperatures. 
data of $\mathrm{T} 2 \mathrm{CNH}$ are well supported by the harmonic and related literature values. The $\beta 0$ value of $\mathrm{T} 2 \mathrm{CNH}$ molecule is found to be $1.5861 \times 10^{-30} \mathrm{esu}$, which is four time greater thatn that of urea. The NBO result reflects the charge transfer with in the molecule and the maximum energy takes place during $\pi-\pi *$ transition. The Homo-Lumo band gap was calculated about $4.025 \mathrm{eV}$, which leads the $\mathrm{T}_{2} \mathrm{CNH}$ molecule to become less stable and more reactive. The recorded UV-Vis spectral values are agree well with calculated values. MEP surface predict the reactive sites for electrophilic and nucleophilic attack. In addition, Mulliken atomic charges, zero point energy and thermodynamic properties are also calculated. 


\section{References}

1 The Encyclopœdia Britannica (1882), Nature 25: 313-315.

2 Molvi KI, Vasu KK, Yerande SG, Sudarsanam V, Haque N (2007) Syntheses of new tetrasubstituted thiophenes as novel antiinflammatory agents. Euro J Medi Chem 42: 1049-1058.

3 Rai NS, Kalluraya B, Lingappa B, Shenoy S, Puranic VG (2008) Convenient access to 1,3,4-trisubstituted pyrazoles carrying 5-nitrothiophene moiety via 1,3-dipolar cycloaddition of sydnones with acetylenic ketones and their antimicrobial evaluation. Euro J Medi Chem 43: 1751-1720.

4 Ashalatha BV, Narayana B, Raj KKV, Kumari NS (2008) Synthesis of some new bioactive 3-amino-2-mercapto-5,6,7,8-tetrahydro[1] benzothieno[2,3-d]pyrimidin-4(3H)-one derivatives. Euro J Medi Chem 42: 719-728.

5 Polivka Z, Holubek J, Svatek E, Metys J, Protiva M (1984) Potential hypnotics and anxiolytics: Synthesis of 2-bromo-4-(2-chlorophenyl)9-[4-(2-methoxyethyl)piperazino]-6H-thieno[3,2,4-triazolo[4,3-a]1,4-diazepine and of some related compounds. Collect Czech Chem Commun 49: 621-623.

6 NoguchiH,KitazumiK, MoriM,ShibaT(2004)Electroencephalographic Properties of Zaleplon, a Non-Benzodiazepine Sedative/Hypnotic, in Rats. J Pharm Sci 94: 246-251.

7 Kim S, Yoon JY (2004) Hydrazones in science and synthesis. Sci Synth 27: 671-722.

8 Brehme R, Enders D, Fernandez R, Lassaletta JM, Aldehyde N (2007) $\mathrm{N}$-Dialkylhydrazones as Neutral Acyl Anion Equivalents: Umpolung of the Imine Reactivity. Eur J Org Chem 34: 5629-5660.

9 Belskaya NP, Dehaen W, Bakulev VA (2010) Synthesis and properties of hydrazones bearing amide, thioamide and amidine functions. Archive Org Chem 275-332.

10 Bhole RP, Bhusari KP (2009) Synthesis, antimycobacterial activity and 3-d qsar studies of some new derivatives of $p$-hydroxybenzohydrazide. QSAR Comb Sci 28: 1405-1417.

11 Loncle C, Brunel JM, Vidal N, Dherbomez M, Letourneux Y (2004) Synthesis and antifungal activity of cholesterol-hydrazone derivatives. Eur J Med Chem 39: 1067-1071.

12 KK Bedia, Elcin O, Seda U, Fatma K, Nathaly S (2006) Synthesis and characterization of novel hydrazide-hydrazones and the study of their structure-antituberculosis activity. Eur J Med Chem 41: 12531261.

13 Subashchandrabose S, Meganathan C, Erdogdu Y, Saleem H, Jayakumar C (2013) Vibrational and conformational analysis on N1N2-bis(pyridine-4-yl)methylene)benzene-1, 2-diamine. J Mol Struct 1042: 37-44.

14 Subramaninan N, Sundaraganesan N, Jayabharathi JA (2010) Molecular structure, spectroscopic (FT-IR, FT-Raman, NMR, UV) studies and first order molecular hyperpolarizabilities of 1,2-bis (3-methoxy-4-hydroxy benzlidene) hydrazine by density functional method. Spectrochim Acta A Mol Biomol Spectrosc 76: 259-269.

15 Frisch MJ, Trucks GW, Schlegel HB, Scuseria GE, Robb MA, et al. (2004) Theoretical and Computational Aspects of Magnetic Organic Molecules. Gaussian Inc, Wallingford, CT.

16 Schlegel HB (1982) Optimization of equilibrium geometries and transition structures. J Comput Chem 3: 214-218.
17 Jamróz MH (2006) Vibrational modes of 2,6-, 2,7-, and 2,3-diisopropylnaphthalene, A DFT study. J Mol Struct 787: 172-183.

18 Michalska D (2003) Raint Program, Wroclaw University of Technology, Poland.

19 Michalska D, Wysokinski R (2005) The prediction of Raman spectra of platinum(II) anticancer drugs by density functional theory. Chem Phys Lett 403: 211-217.

20 Fleming GD, Koch R, Vallete MMC (2006) Theoretical study of the syn and anti thiophene-2-aldehyde conformers using density functional theory and normal coordinate analysis. Spectrochim Acta A Mol Biomol Spectrosc 65: 935-945.

21 Balachandran V, Janaki A, Nataraj A (2014) Theoretical investigations on molecular structure, vibrational spectra, HOMO, LUMO, NBO analysis and hyperpolarizability calculations of thiophene-2carbohydrazide. Spectrochim Acta A Mol Biomol Spectrosc 118: 321-330.

22 Unal A, Eren B (2013) FT-IR, dispersive Raman, NMR, DFT and antimicrobial activity studies on 2-(Thiophen-2-yl)-1H-benzo[d] imidazole. Spectrochim Acta A Mol Biomol Spectrosc 114: 129-136.

23 Brathen O, Kveseth K, Nielsen CJ, Hagen K (1986) Molecular structure and conformational equilibrium of gaseous thiophene-2-aldehyde as studied by electron diffraction and microwave, infrared, Raman and matrix isolation spectroscopy. J Mol Struct 145: 45-68.

24 Geiger DK, Geiger HC, Williams L, Noll BC (2012) 2-(Thiophen-2-yl)1-(thiophen-2-ylmethyl)-1 H -benzimidazole. Acta Cryst E: Structure Reports Online 68: 0420.

25 Rauhut G, Pulay P (1995) Transferable Scaling Factors for Density Functional Derived Vibrational Force Fields. J Phys Chem 99: 30933100.

26 Bellamy $\sqcup$ (1980) The infrared spectra of complex molecules, vol 2 champman and Hall, London.

27 Babu NR, Subashchandrabose S, Padusha MSA, Saleem H, Manivannan $\mathrm{V}$, et al. (2014) Synthesis and structural characterization of (E)- $\mathrm{N}^{\prime}$ ((Pyridin-2-yl) methylene) benzohydrazide by X-ray diffraction, FTIR, FT-Raman and DFT methods. J Mol Struct 1072: 84-93.

28 Varsanyi G (1974) Assignments of vibrational spectra of 700 Benzene Derivatives, Wiley, New York.

29 Sathyanarayanan DN (2000) Vibrational spectroscopy theory and Applications, 446-447.

30 Varsanyi G (1973) Assignments for Vibrational Spectra of Seven Hundred Benzene derivatives, 1/2 Academic Kiaclo.

31 Jag M (2001) Organic Spectroscopy Principles and Application, (2nd edn), Narosa Publishing house, New Delhi.

32 Lorenc J (2012) Dimeric structure and hydrogen bonds in 2-N-ethylamino-5-metyl-4-nitro-pyridine studied by XRD, IR and Raman methods and DFT calculations. Vib Spectrosc 61: 112-123.

33 Krishnakumar V, Dheivamalar S, Xavier RJ, Balachandran V (2006) Analysis of vibrational spectra of 4-amino-2, 6-dichloropyridine and 2-chloro-3, 5-dinitropyridine based on density functional theory calculations. Spectrochim Acta A Mol Biomol Spectrosc 65: 147-154.

34 Sundaraganesan N, Saleem H, Mohan S (2003) Vibrational spectra, assignments and normal coordinate analysis of 2-amino-5bromopyridine. Spectrochim Acta A Mol Biomol Spectrosc 59: 11131118.

35 Silverstein M, Basseller CG, Morill C (1981) Spectrometric identification of organic compound, Wiley; \. 
36 Badawi HM (2009) Structural stability, C-N internal rotations and vibrational spectral analysis of non-planar phenylurea and phenylthiourea. Spectrochim Acta A Mol Biomol Spectrosc 72: 523527.

37 James C, Ravikumar C, Sundius T, Krishnakumar V, Kesavamoorthy R, et al. (2008) FT-Raman and FTIR spectra, normal coordinate analysis and ab initio computations of (2-methylphenoxy) acetic acid dimer. Vib Spectrosc 47: 10-20.

38 Weinhold F, Landis CR (2005) Valency and bonding: a natural bond orbital donor-acceptor perspective. Cambridge University Press.

39 Ott JB, Boerio-Goates J (2000) Calculations from Statistical Thermodynamics. 